\title{
SINGULAR LIMIT ANALYSIS OF PLANAR EQUILIBRIUM SOLUTIONS TO A CHEMOTAXIS MODEL EQUATION WITH GROWTH
}

\author{
Tohru Tsujikawa
}

\begin{abstract}
We consider the stability of planar localized equilibrium solutions of a chemotaxis model equation with growth term, which describes aggregation of biological individuals. Assuming that the diffusion and chemotactic rates are both very small compared with the growth rate, we introduce a small parameter, say $\varepsilon$, into the equation. The asymptotic form of the eigenvalues with respect to the parameter, which is essential to determine the stability for the linearized equation, can be obtained by using singular limit analysis as $\varepsilon \downarrow 0$.
\end{abstract}

\section{Introduction}

It is known that the movement of some biological individuals is affected by an acute sense of smell which conveys information between individuals or groups. One typical example is chemotactic movement which is the process by which biological individuals aggregate by moving preferentially toward higher concentrations of chemotactic substances [1,6]. Recently, Budrene and Berg [3] have shown experimentally that the bacterium $E$. coli exhibits complex two-dimensional spots or stripe patterns by the interplay of diffusion, growth, and aggregation in response to the gradients of attractant (aspartte). To analyze the spatial pattern formation caused by the interdependence of these characteristics, we propose the following chemotaxis model equations with growth term:

$$
\begin{aligned}
& \frac{\partial u}{\partial \tau}=d_{u} \Delta u-\delta \nabla(u \cdot \nabla \chi(v))+f(u) \\
& \frac{\partial v}{\partial \tau}=d_{v} \Delta v+\beta u-\gamma v
\end{aligned}
$$

where $u(\tau, \mathbf{x})$ and $v(\tau, \mathbf{x})$ are, respectively, the population density and the concentration of chemotactic substance at time $\tau$ and position $\mathbf{x} \in \Omega \subset \mathbf{R}^{2} . \Delta$ and $\nabla$ are the Laplace and the gradient operators with respect to $\mathbf{x} . d_{u}$ and $d_{v}$ are the diffusion rates of $u$ and $v$, respectively, and $\beta$ and $\gamma$ are the production and degradation rates. $\delta \nabla \chi(v)$ is the velocity of the direct movement of $u$ due to chemotaxis, which generally satisfies $\chi^{\prime}(v)>0$ for $v>0$. Ford and Lauffenburger [8] summarized some plausible functional forms of $\chi(v)$. In the absence of the growth term $f(u),(1.1)$ is called the Keller-Segel model [13], which describes slime mold aggregation. As for the growth term $f(u)$, several forms are proposed (see Murray [18], for instance). Here we consider the case of the bistable type, $f(0)=f(\underline{u})=f(\bar{u})=0$ for some $0<\underline{u}<\bar{u}$, $f(u)<0$ for $0<u<\underline{u}, f(u)>0$ for $\underline{u}<u<\bar{u}$, and $f^{\prime}(0)<0, f^{\prime}(\bar{u})<0$. This includes the effects of intraspecific competition and cooperation.

Received April 10, 1995, revised January 30, 1996.

Key words and phrases: chemotaxis, planar equilibrium solution, stability, singular perturbation. 
From the biological view point [2, 3], we are concerned with (1.1) when (i) the migration of individuals by diffusion and chemotaxis is slow compared with the diffusivity of the chemotactic substance and (ii) the movement of individuals is mainly due to chemotaxis. We thus assume

$$
\frac{d_{u}}{d_{v}} \ll \frac{\delta}{d_{v}} \ll 1
$$

and that there is a small parameter $\varepsilon$ such that

$$
\varepsilon^{2}=\frac{d_{u}}{d_{v}} \quad \text { and } \quad \varepsilon k=\frac{\delta}{d_{v}}
$$

with some positive constant $k$.

By using a suitable transformation, the equations (1.1) can be rewritten as

$$
\begin{aligned}
& \frac{\partial u}{\partial \tau}=\varepsilon^{2} \Delta u-\varepsilon k \nabla(u \cdot \nabla \chi(v))+f(u) \\
& \frac{\partial v}{\partial \tau}=\Delta v+u-\gamma v
\end{aligned}
$$

where $f(u)$ satisfies $f(0)=f(a)=f(1)=0(0<a<1)$.

In this paper, we consider (1.2) in the strip domain $\Omega=\Omega_{\ell}=\mathbf{R} \times(0, \ell) \subset \mathbf{R}^{2}$. Then the corresponding boundary and initial conditions for (1.2) are

$$
\begin{array}{ll}
\lim _{|x| \rightarrow \infty}(u(\tau, x, y), v(\tau, x, y))=(0,0) & (\tau, y) \in \mathbf{R}_{+} \times(0, \ell), \\
\frac{\partial}{\partial y} u(\tau, x, 0)=0=\frac{\partial}{\partial y} u(\tau, x, \ell), & (\tau, x) \in \mathbf{R}_{+} \times \mathbf{R}, \\
\frac{\partial}{\partial y} v(\tau, x, 0)=0=\frac{\partial}{\partial y} v(\tau, x, \ell), &
\end{array}
$$

and

$$
(u(0, x, y), v(0, x, y))=\left(u_{0}(x, y), v_{0}(x, y)\right), \quad(x, y) \in \Omega_{\ell},
$$

where $\lim _{|x| \rightarrow \infty}\left(u_{0}(x, y), v_{0}(x, y)\right)=(0,0)$. Recently, Furuya and Yagi [25] have shown the existence of the global solutions of (1.2)-(1.5) when the space dimensions $n=1,2$.

When $\int_{0}^{1} f(u) d u>0$, by using an approximate equation of (1.2)-(1.5) as the limit $\varepsilon \downarrow 0$, which we call a limiting system (see (2.5)), it is shown in [15] that there is a 1-dimensional symmetric localized equilibrium solution to (1.2), (1.3), (1.5), which means an aggregating pattern, due to the suitable balance among diffusion, chemotaxis, and growth. The 1-dimensional equilibrium solution can be extended uniformly in the $y$-direction in $\Omega_{\ell}$, and it is obvious that this solution is also an equilibrium solution of (1.2)-(1.5) in $\Omega_{\ell}$, which we call a planar equilibrium solution in $\Omega_{\ell}$. We study the stability of the planar equilibrium solutions depending on the form of $\chi(v)$ as well as on $\ell>0$ and $k>0$. It is numerically shown in [15] that, for $\chi(v)=v$, the solution is always stable for any $\ell>0$, and for $\chi(v)=s v^{2} /\left(s+v^{2}\right)$, its stability changes depending on the values of $\ell>0, k>0$, and $s>0$.

Motivated by these numerical results, we study the stability of planar equilibrium solutions of (1.2)-(1.5) for sufficiently small $\varepsilon>0$.

Let us state the definition of the stability of the planar equilibrium solution of (1.2)-(1.5) in $\Omega_{\ell}$.

Definition. The planar equilibrium solution of (1.2)-(1.5) is stable with shift if and only if the spectrum of the linearized operator around the solution contains a simple zero eigenvalue and the remaining spectrum is contained in a closed angle lying in 
the left half of the complex plane (see [9, 22], for instance). Unless it has a nonzero spectrum point with nonnegative real part, the solution is unstable.

The aim of this paper is to determine the distribution of eigenvalues since they play an essential role for the stability of the equilibrium solution. We first show that eigenvalues which do not satisfy the condition (5.7) are all in the left half of the complex plane and away from the imaginary axis (Proposition 1). Therefore, it is important for stability to show the distribution of the other eigenvalues, which we call critical eigenvalues. To do that, we consider the limiting system of (1.2)-(1.5) as $\varepsilon \downarrow 0$.

In Section 2, we introduce the limiting system which is derived from (1.2)-(1.5) as $\varepsilon \downarrow 0$. In Section 3, the existence of 1-dimensional symmetric equilibrium solutions of (1.2)-(1.5) is proved for sufficiently small $\varepsilon>0$ (Theorem 1) by using singular perturbation methods. From the limiting system (4.1) as $\varepsilon \downarrow 0$, in Section 4, we obtain the ordinary differential equations (4.2) which the Fourier components of the disturbances for the planar equilibrium solution satisfy. In Section 5 , by using the SLEP method [19, 24], we show that the asymptotic form of critical eigenvalues of the linearized problem of (1.2)-(1.5) around the planar equilibrium solution equals the coefficients of the ordinary differential equations which are obtained in Section 4 (Theorem 2). Though we should say that the planar equilibrium solution of (1.2)-(1.5) is stable with respect to discrete spectrum if all eigenvalues of the linearized problem have negative real part except for a simple zero eigenvalue, we simply say that it is stable. It follows from Theorem 2 that when the width $\ell>0$ of the strip domain becomes $O\left(\varepsilon^{\frac{1}{2}}\right)$ for sufficiently small $\varepsilon>0$, the stability is determined by that of the 1-dimensional equilibrium solutions for the limiting system in Section 6 (Corollary 2). On the other hand, if $\ell>0$ is suitably large, the stability of 1-dimensional solutions is not necessarily inherited by the planar solutions, which depend on the form of $\chi(v)$ as well as the value of $k>0$.

Some function spaces are needed in this paper:

$$
\begin{aligned}
& H_{N}^{n}\left(\mathbf{R}_{+}\right) \equiv\left\{u \in H^{n}\left(\mathbf{R}_{+}\right) \mid u_{x}(0)=0\right\}, \\
& L_{\rho}^{2}(\Omega) \equiv\left\{u \in L^{2}(\Omega) \mid\|u\|_{L_{\rho}^{2}} \equiv\left(\int_{\Omega}\left|e^{\rho|x|} u(x)\right|^{2} d x\right)^{1 / 2}<+\infty\right\}, \\
& H^{-1}(\Omega) \equiv \text { the dual space of } H^{1}(\Omega),
\end{aligned}
$$

and $C_{c . u .}^{n}(\Omega)$-sense means the uniform convergence on any compact subset of $\Omega$ with respect to $C^{n}(\Omega)$-norm where $\mathbf{R}_{+}=(0, \infty), \rho$ is a positive number, $n$ is a nonnegative integer, and $H^{n}(\Omega)$ is the usual Sobolev space on $\Omega$.

\section{Limiting system as $\varepsilon \downarrow 0$}

In this section, following [15], we formally introduce the limiting system as $\varepsilon \downarrow 0$ for (1.2)-(1.5) in $\Omega_{\ell}$, which gives the essential information for the existence and stability of symmetric localized equilibrium solutions. To do that, we use the transformation $t=\varepsilon \tau$ for (1.2). Then (1.2) is rewritten as

$$
\begin{aligned}
& \frac{\partial u}{\partial t}=\varepsilon \Delta u-k \nabla(u \cdot \nabla \chi(v))+\frac{1}{\varepsilon} f(u), \\
& \varepsilon \frac{\partial v}{\partial t}=\Delta v+u-\gamma v .
\end{aligned}
$$

If initial data are smooth, both the diffusion and chemotaxis terms of the first equation in (2.1) are small in comparison with $\frac{1}{\varepsilon} f(u)$, that is, the two terms may be neglected 
for a short time so that the first equation of (2.1) can be approximated by the ordinary differential equation $u_{t}=\frac{1}{\varepsilon} f(u)$. Therefore, $u(t, x, y)$ tends quickly to either 0 or 1 . It turns out that after a short time, the space is partitioned into three regions: a region $\Omega_{0}^{\varepsilon}$ where $u$ is almost equal to 0 , a region $\Omega_{1}^{\varepsilon}$ where $u$ is almost equal to 1 , and a "thin" strip region $R^{\varepsilon}$ which links $\Omega_{0}^{\varepsilon}$ and $\Omega_{1}^{\varepsilon}$. As $\varepsilon$ tends to zero, $\Omega_{0}^{\varepsilon}$ and $\Omega_{1}^{\varepsilon}$ become formally $\Omega_{0}$ and $\Omega_{1}$ where the value of $u$ is 0 in $\Omega_{0}$ and 1 in $\Omega_{1}$ and the region $R^{\varepsilon}$ becomes a 1-dimensional curve $\Gamma(t)$, which we call the interface (see Chen [4], for instance). Subsequently, the diffusion and chemotaxis terms of $u$ in (2.1) still can be neglected, and therefore $u(t, x, y)$ approximately equals 0 or 1 depending on the region away from the interface, whereas the dynamics of $v$ is approximately obtained by solving

$$
\begin{array}{ll}
0=\Delta v+g_{i}(v), & t>0, \quad(x, y) \in \Omega_{i}(t) \quad(i=0,1), \\
\lim _{|x| \rightarrow \infty} v(t, x, y)=0, & t>0, \quad y \in(0, \ell), \\
\frac{\partial}{\partial y} v(t, x, 0)=0=\frac{\partial}{\partial y} v(t, x, \ell), & t>0, \quad x \in \mathbf{R},
\end{array}
$$

where $g_{0}(v)=-\gamma v, g_{1}(v)=1-\gamma v$, and $\Omega_{i}(t)=\left\{(x, y) \in \Omega_{\ell} \mid u(t, x, y)=i\right\}$. On the other hand, near the interface, a layer develops, so that the diffusion and chemotaxis terms are no longer negligible. Then these two terms will become large enough to balance approximately the reaction term $\frac{1}{\varepsilon} f(u)$, and the difference of the magnitudes of each term is a driving force which makes the interface move. The motion of the interface will be determined by the equation

$$
\Gamma_{t}=\left\{c+k \chi^{\prime}(v) \cdot \frac{\partial v}{\partial N}-\varepsilon(n-1) \kappa\right\} \cdot N, \quad t>0, \quad(x, y) \in \Gamma(t),
$$

where $N$ is the outward unit normal vector on $\Gamma(t)$ and $\kappa$ is the mean curvature at the interface (for the formal derivation, see [15]). Here, $c$ is the velocity of the traveling front solution $w(x-c t)$ of the system

$$
\begin{aligned}
& w_{t}=w_{x x}+f(w), \quad t>0, \quad x \in \mathbf{R}, \\
& w(t,-\infty)=1, \quad w(t, \infty)=0, \quad t>0,
\end{aligned}
$$

for which the velocity $c$ is uniquely determined where $c \geq 0$ (resp., $<0$ ) if $\int_{0}^{1} f(u) d u \geq$ 0 (resp., < 0 ). Moreover, if the initial interface is orthogonal to the boundary, then the interface moves so as to intersect the boundary orthogonally (see [4]), that is,

$$
N \cdot N_{\Omega}=0, \quad t>0, \quad(x, y) \in \Gamma(t) \cap \partial \Omega_{\ell},
$$

where $N_{\Omega}$ means the outward unit normal vector on $\partial \Omega_{\ell}$. Integrating (2.2) and (2.3), we have

$$
\begin{aligned}
& \Gamma_{t}=\left\{c+k \chi^{\prime}(v) \cdot \frac{\partial v}{\partial N}-\varepsilon \kappa\right\} \cdot N, \quad t>0, \quad(x, y) \in \Gamma(t), \\
& N \cdot N_{\Omega}=0, \quad t>0, \quad(x, y) \in \Gamma(t) \cap \partial \Omega_{\ell}, \\
& 0=\Delta v+g_{i}(v), \quad t>0, \quad(x, y) \in \Omega_{i}(t), \\
& \lim _{|x| \rightarrow \infty} v(t, x, y)=0, \quad t>0, \quad y \in(0, \ell), \\
& \frac{\partial}{\partial y} v(t, x, 0)=0=\frac{\partial}{\partial y} v(t, x, \ell), \quad t>0, \quad x \in \mathbf{R} .
\end{aligned}
$$

The smoothness of $v$ on the interface $\Gamma(t)$ should be $C^{1}$, that is,

$$
v(t, \cdot) \in C^{1}\left(\Omega_{\ell}\right), \quad t>0 .
$$


We call (2.5) the limiting system or simply the interface equation of (2.1).

By solving this limiting system, the approximate solutions corresponding to the planar symmetric equilibrium solution of (1.2)-(1.5) for sufficiently small $\varepsilon>0$ are constructed in the next section. Using this approximate solution, we have the planar equilibrium solution of (1.2)-(1.5) for sufficiently small $\varepsilon>0$.

\section{Existence of the 1-dimensional symmetric equilibrium solutions}

In order to obtain planar symmetric equilibrium solutions, we only show the existence of 1-dimensional localized equilibrium solutions of (1.2)-(1.5) which are symmetric at $x=0$. To do that, we first consider the 1-dimensional stationary problem corresponding to (2.5) in $\mathbf{R}_{+}$. Put $\Omega_{0}=\left\{x \in \mathbf{R}_{+} \mid x>\eta\right\}$ and $\Omega_{1}=\left\{x \in \mathbf{R}_{+} \mid x<\eta\right\}$ with an interface point $\eta \in(0, \infty)$. The resulting problem is to find $(\eta, v(x))$ which satisfies

$$
\begin{aligned}
& 0=c+k \chi^{\prime}(v) v_{x}, \quad x=\eta, \\
& 0=v_{x x}+g_{i}(v), \quad x \in \Omega_{i} \quad(i=0,1), \\
& v_{x}(0)=0, \quad \lim _{x \rightarrow \infty} v(x)=0, \quad \text { and } \quad v \in C^{\mathbf{1}}\left(\mathbf{R}_{+}\right) .
\end{aligned}
$$

We first fix $\eta>0$ arbitrarily. Then the solution $v(x ; \eta)$ of the second and third equations of (3.1) is described by

$$
v(x ; \eta) \equiv \begin{cases}\frac{1}{\gamma}+\left(\sigma-\frac{1}{\gamma}\right) \frac{\left(e^{\sqrt{\gamma} x}+e^{-\sqrt{\gamma} x}\right) e^{\sqrt{\gamma} \eta}}{1+e^{2 \sqrt{\gamma} \eta}}, & x \in(0, \eta), \\ \sigma e^{-\sqrt{\gamma}(x-\eta)}, & x \in(\eta, \infty),\end{cases}
$$

with

$$
\sigma=\frac{1-e^{-2 \sqrt{\gamma} \eta}}{2 \gamma}
$$

By substituting (3.2) in the first equation of (3.1), the solution $(\eta, v(x ; \eta))$ of $(3.1)$ is given by solving

$$
H(\eta) \equiv c-\frac{k\left(1-e^{-2 \sqrt{\gamma} \eta}\right)}{2 \sqrt{\gamma}} \chi^{\prime}\left(\frac{1-e^{-2 \sqrt{\gamma} \eta}}{2 \gamma}\right)=0 .
$$

If there is an $\eta^{*}>0$ satisfying $H\left(\eta^{*}\right)=0$, a solution of (3.1) is represented as $\left(\eta^{*}, v\left(x ; \eta^{*}\right)\right)$.

\section{Assumption 1.}

$$
\chi^{\prime}(v)>0 \quad \text { for } \quad v>0 .
$$

Under this assumption, it has been shown that if $c \leq 0$, then there is no solution of (3.3), and if $c>0$, then there is a $k^{*}>0$ satisfying $c-\frac{k^{*}}{2 \sqrt{\gamma}} \chi^{\prime}\left(\frac{1}{2 \gamma}\right)=0$ such that there is at least one solution $\left(\eta^{*}, v\left(x ; \eta^{*}\right)\right)$ of $(3.1)$ for any $k>k^{*}$. In fact, there is a specific form $\chi(v)$ for which many solutions of (3.1) coexist (see [15]).

We thus assume

\section{Assumption 2.}

$$
c>0 \quad\left(\text { or } \quad \int_{0}^{1} f(u) d u>0\right)
$$


As a simple but suggestive form of $f(u)$, we take $f(u)=u(1-u)(u-a)$, in which case Assumption 2 requires $0<a<1 / 2$.

When $\chi(v)=v$ and $\chi(v)=s v^{2} /\left(s+v^{2}\right)$, the global structures of $H(\eta)=0$ are drawn in Figures 1 and 2.

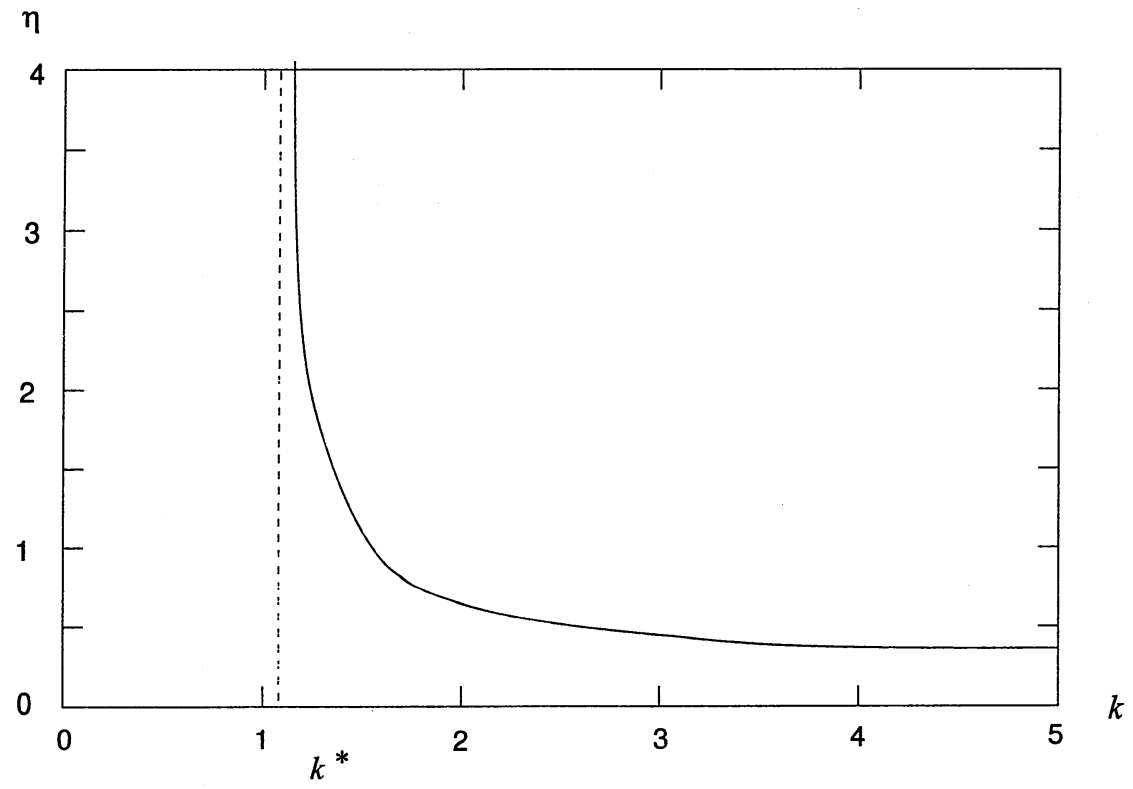

FIGURE 1. Dependency of $k$ on solutions of (3.3) for $\chi(v)=v$, $a=0.1$, and $\gamma=1.0$.

Let $\eta^{*}>0$ be a solution of $H(\eta)=0$ satisfying

$$
\frac{\partial}{\partial \eta} H\left(\eta^{*}\right) \neq 0
$$

and define the pair of functions $\left(u^{0}(x), v^{0}(x)\right)$ by

$$
\begin{aligned}
& u^{0}(x)= \begin{cases}1 & \text { for }|x|<\eta^{*} \\
0 & \text { for }|x|>\eta^{*}\end{cases} \\
& v^{0}(x)= \begin{cases}v\left(x ; \eta^{*}\right) & \text { for } 0<x, \\
v\left(-x ; \eta^{*}\right) & \text { for } 0>x,\end{cases}
\end{aligned}
$$

which is called a 1-dimensional symmetric equilibrium solution $\left(u^{0}(x), v^{0}(x)\right)$ of $(3.1)$ in the limit $\varepsilon \downarrow 0$.

By using $\left(u^{0}(x), v^{0}(x)\right)$, we construct the 1-dimensional localized symmetric equilibrium solution of the problem (1.2)-(1.5) for sufficiently small $\varepsilon>0$. The equilibrium solution $(u(x), v(x))$ which is symmetric at $x=0$ satisfies

$$
\begin{aligned}
& 0=\varepsilon^{2} u_{x x}-\varepsilon k\left\{u \chi^{\prime}(v) v_{x}\right\}_{x}+f(u), \quad x \in(0, \infty), \\
& 0=v_{x x}+u-\gamma v,
\end{aligned}
$$

with

$$
\left(u_{x}(0), v_{x}(0)\right)=(0,0) \quad \text { and } \quad \lim _{x \rightarrow \infty}(u(x), v(x))=(0,0)
$$



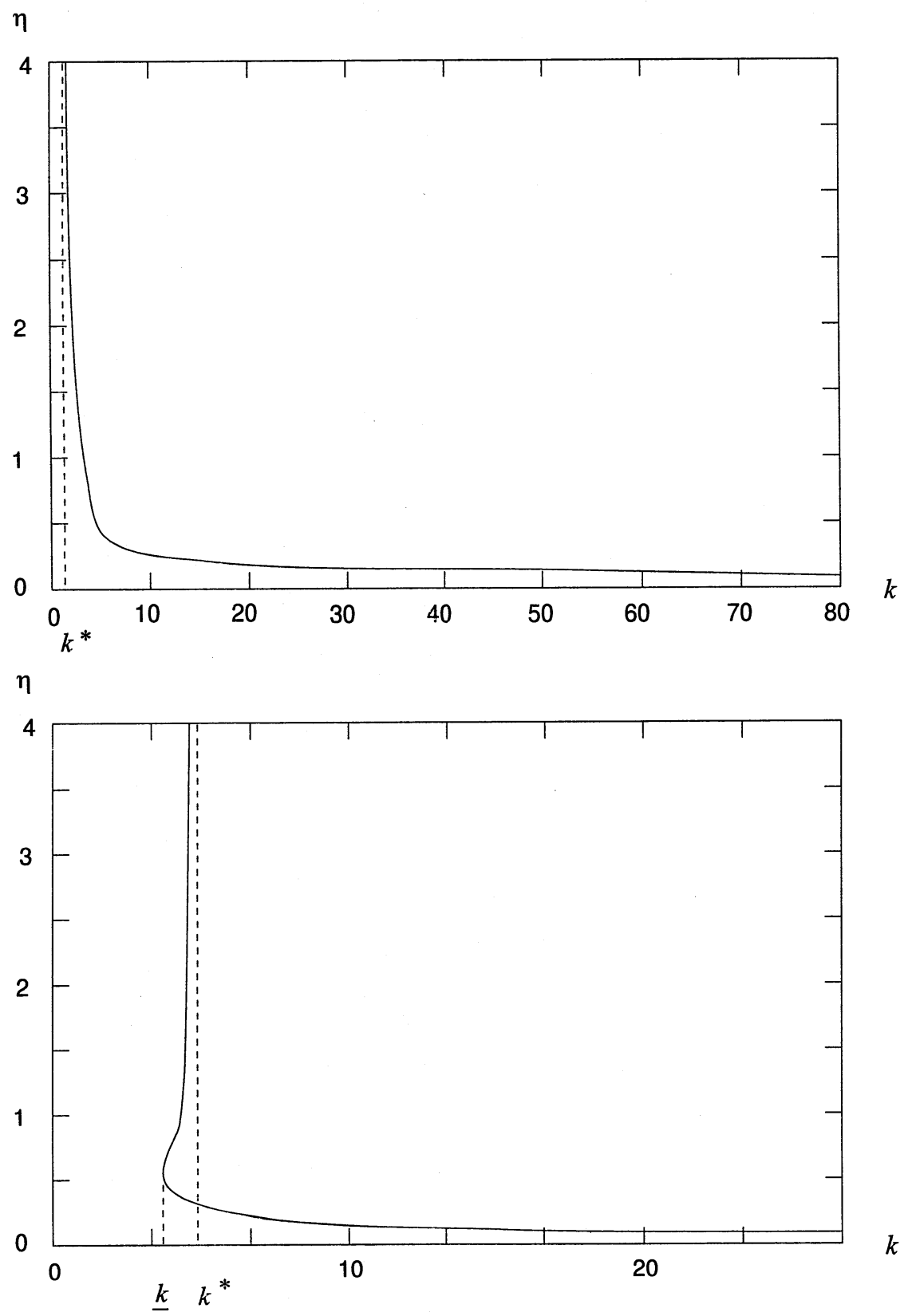

FIGURE 2. Dependency of $k$ on solutions of (3.3) for $\chi(v)=s v^{2} /(s+$ $\left.v^{2}\right)$. Other parameters are as in Figure 1. The global structures are different depending on the value of $s>0$ : (i) $1 / 4 \gamma<s(s=3.0)$; (ii) $0<s<1 / 4 \gamma^{2}(s=0.1)$.

Using the singular perturbation method [7, 14], we obtain

Theorem 1. Under Assumptions 1 and 2, there is an $\varepsilon_{0}>0$ such that for any $\varepsilon \in\left(0, \varepsilon_{0}\right)$ a 1-dimensional equilibrium solution $\left(u^{\varepsilon}(x), v^{\varepsilon}(x)\right)$ of $(3.6)$, (3.7) exists 
and satisfies

$$
\begin{aligned}
& \lim _{\varepsilon \downarrow 0} u^{\varepsilon}(x)=u^{0}(x) \quad \text { uniformly in }[0, \eta(\varepsilon)-\delta) \cup(\eta(\varepsilon)+\delta, \infty), \\
& \lim _{\varepsilon \downarrow 0} v^{\varepsilon}(x)=v^{0}(x) \quad \text { uniformly in } \quad[0, \infty)
\end{aligned}
$$

with a small constant $\delta>0$ where $\eta(\varepsilon)$ satisfies $\lim _{\varepsilon \downarrow 0} \eta(\varepsilon)=\eta^{*}$. Moreover, for $\tilde{u}^{\varepsilon}(\xi)=u^{\varepsilon}(\eta(\varepsilon)+\varepsilon \xi)$ and $\tilde{v}^{\varepsilon}(\xi)=v^{\varepsilon}(\eta(\varepsilon)+\varepsilon \xi)$,

$$
\begin{aligned}
& \lim _{\varepsilon \downarrow 0} \tilde{u}^{\varepsilon}(\xi)=W_{0}(\xi) \quad \text { in } C_{c . u}^{2}(\mathbf{R}) \text {-sense } \\
& \lim _{\varepsilon \downarrow 0} \tilde{v}^{\varepsilon}(\xi)=\sigma^{*} \quad \text { in } C_{c . u}^{2}(\mathbf{R}) \text {-sense }
\end{aligned}
$$

where $W_{0}(\xi)$ is the traveling front solution of (2.4) with velocity $c$.

The proof is given in Section 7.

This theorem shows that $\left(u^{0}(x), v^{0}(x)\right)$ and $\left(W_{0}(\xi), \sigma^{*}\right)$ are good approximate solutions corresponding to the equilibrium ones of (3.6), (3.7). We thus can construct the 1-dimensional symmetric equilibrium solution $\left(u^{\varepsilon}(x), v^{\varepsilon}(x)\right)$ of the stationary problem of (1.2)-(1.4) by

$$
\left(u^{\varepsilon}(x), v^{\varepsilon}(x)\right)= \begin{cases}\left(u^{\varepsilon}(x), v^{\varepsilon}(x)\right) & \text { for } x \geq 0 \\ \left(u^{\varepsilon}(-x), v^{\varepsilon}(-x)\right) & \text { for } x<0\end{cases}
$$

We define the planar equilibrium solutions $\left(u^{\varepsilon}(x, y), v^{\varepsilon}(x, y)\right)$ of $(1.2)-(1.4)$ by extending $\left(u^{\varepsilon}(x), v^{\varepsilon}(x)\right)$ uniformly in the $y$-direction in the strip domain $\Omega_{\ell}=\mathbf{R} \times(0, \ell)$ and similarly the planar equilibrium solution $\left(u^{0}(x, y), v^{0}(x, y)\right)$ of the limiting system by extending $\left(u^{0}(x), v^{0}(x)\right)$. We note that the latter solution is characterized by the interface positions $\left\{\left( \pm \eta^{*}, y\right) \mid y \in(0, \ell)\right\}$ and $v^{0}(x, y)$.

We now consider the distribution of the eigenvalues of the linearized problem around $\left(u^{\varepsilon}(x, y), v^{\varepsilon}(x, y)\right)$ for sufficiently small $\varepsilon>0$. Before doing that, we study the eigenvalue problem around $\left(u^{0}(x, y), v^{0}(x, y)\right)$ in the limiting system as $\varepsilon \downarrow 0$.

\section{Singular limit eigenvalue problem}

In this section, we consider the linearized eigenvalue problem of the limiting system (2.5) according to the argument used in the previous paper [15]. Let us consider the situation when the interface position $\Gamma_{ \pm}^{0}=\left\{\left( \pm \eta^{*}, y\right) \mid y \in(0, \ell)\right\}$ is perturbed to become $\Gamma_{ \pm}(0)=\left\{\left( \pm \eta^{*}+\zeta_{0}^{ \pm}(y), y\right) \mid y \in(0, \ell)\right\}$. From (2.5), the time-evolution of the interface curves $\Gamma_{ \pm}(t)=\left\{\left( \pm \eta^{*}+\zeta^{ \pm}(t, y), y\right) \mid y \in(0, \ell)\right\}$ is described by

$$
\begin{aligned}
& \frac{\partial \zeta^{+}}{\partial t}=c \sqrt{1+\left(\frac{\partial \zeta^{+}}{\partial y}\right)^{2}}+k \chi^{\prime}(v)\left[\frac{\partial v}{\partial x}-\frac{\partial v}{\partial y} \frac{\partial \zeta^{+}}{\partial y}\right]+\frac{\varepsilon \zeta_{y y}^{+}}{1+\left(\frac{\partial \zeta^{+}}{\partial y}\right)^{2}}+R^{+} \\
& \frac{\partial \zeta^{-}}{\partial t}=-c \sqrt{1+\left(\frac{\partial \zeta^{-}}{\partial y}\right)^{2}}+k \chi^{\prime}(v)\left[\frac{\partial v}{\partial x}-\frac{\partial v}{\partial y} \frac{\partial \zeta^{-}}{\partial y}\right]-\frac{\varepsilon \eta_{y y}^{-}}{1+\left(\frac{\partial \zeta^{-}}{\partial y}\right)^{2}}+R^{-}
\end{aligned}
$$




$$
\begin{array}{ll}
\left(\zeta^{-}(0, y), \zeta^{+}(0, y)\right)=\left(\zeta_{0}^{-}(y), \zeta_{0}^{+}(y)\right), & y \in(0, \ell), \\
\frac{\partial}{\partial y} \zeta^{-}(t, 0)=0=\frac{\partial}{\partial y} \zeta^{-}(t, \ell), & t>0, \\
\frac{\partial}{\partial y} \zeta^{+}(t, 0)=0=\frac{\partial}{\partial y} \zeta^{+}(t, \ell), & t>0, \\
0=\Delta v+g_{i}(v), & t>0, \quad(x, y) \in \Omega_{i}(t) \quad(i=0,1), \\
\lim _{|x| \rightarrow \infty} v(t, x, y)=0, & t>0, \quad y \in(0, \ell), \\
\frac{\partial}{\partial y} v(t, x, 0)=0=\frac{\partial}{\partial y} v(t, x, \ell), & t>0, \quad x \in \mathbf{R}, \\
v(t, \cdot) \in C^{1}\left(\Omega_{\ell}\right), & t>0,
\end{array}
$$

where $\Omega_{1}(0)=\left\{(x, y) \mid-\eta^{*}+\zeta_{0}^{-}(y)<x<\eta^{*}+\zeta_{0}^{+}(y)\right.$ for $\left.y \in(0, \ell)\right\}, \Omega_{0}(0)=$ $\Omega_{\ell} \backslash \Omega_{1}(0)$, and $R^{ \pm}$are the higher-order terms with respect to $\zeta^{ \pm}$and their derivatives. Suppose that $\zeta^{ \pm}$are given. We can solve (4.1) for $v$. Substituting the solution $v$ into the equations for $\zeta^{ \pm}$, we obtain the equations of $\zeta^{ \pm}$only. Let $\zeta_{\kappa}^{ \pm}(t)$ be the Fourier coefficients of $\zeta^{ \pm}(t)$, which are defined by $\zeta_{\kappa}^{ \pm}(t)=\int_{-\infty}^{\infty} \zeta^{ \pm}(t, y) e^{-i \kappa y} d y$ where $\kappa=m \pi / \ell$ with positive integer $m$. Then the linear parts of the resulting equations for $\zeta^{ \pm}(t, y)$ become

$$
\begin{aligned}
\frac{d \zeta_{\kappa}^{+}}{d t}=- & \varepsilon \kappa^{2} \zeta_{\kappa}^{+}-k \chi^{\prime}\left(\sigma^{*}\right)\left[\frac{e^{-2 \sqrt{\gamma} \eta^{*}}}{2} \zeta_{\kappa}^{+}-\frac{e^{-2 \sqrt{\gamma+\kappa^{2}} \eta^{*}}}{2} \zeta_{\kappa}^{-}\right] \\
& +k \chi^{\prime \prime}\left(\sigma^{*}\right) \frac{1-e^{-2 \sqrt{\gamma} \eta^{*}}}{2 \sqrt{\gamma}}\left[\left(\frac{1-e^{-2 \sqrt{\gamma} \eta^{*}}}{2 \sqrt{\gamma}}-\frac{1}{2 \sqrt{\gamma+\kappa^{2}}}\right) \zeta_{\kappa}^{+}+\frac{e^{-2 \sqrt{\gamma+\kappa^{2}} \eta^{*}}}{2 \sqrt{\gamma+\kappa^{2}}} \zeta_{\kappa}^{-}\right]
\end{aligned}
$$

and

$$
\begin{aligned}
\frac{d \zeta_{\kappa}^{-}}{d t}=- & \varepsilon \kappa^{2} \zeta_{\kappa}^{-}-k \chi^{\prime}\left(\sigma^{*}\right)\left[\frac{e^{-2 \sqrt{\gamma} \eta^{*}}}{2} \zeta_{\kappa}^{-}-\frac{e^{-2 \sqrt{\gamma+\kappa^{2}} \eta^{*}}}{2} \zeta_{\kappa}^{+}\right] \\
& +k \chi^{\prime \prime}\left(\sigma^{*}\right) \frac{1-e^{-2 \sqrt{\gamma} \eta^{*}}}{2 \sqrt{\gamma}}\left[\left(\frac{1-e^{-2 \sqrt{\gamma} \eta^{*}}}{2 \sqrt{\gamma}}-\frac{1}{2 \sqrt{\gamma+\kappa^{2}}}\right) \zeta_{\kappa}^{-}+\frac{e^{-2 \sqrt{\gamma+\kappa^{2}} \eta^{*}}}{2 \sqrt{\gamma+\kappa^{2}}} \zeta_{\kappa}^{+}\right] .
\end{aligned}
$$

We note that disturbances are divided into two types, that is, varicose ones $\left(\zeta_{0}^{+}(y)=\right.$ $\left.-\zeta_{0}^{-}(y)\right)$ and zig-zag ones $\left(\zeta_{0}^{+}(y)=\zeta_{0}^{-}(y)\right)$. Since the corresponding solutions satisfy $\zeta_{\kappa}^{+}(t)=-\zeta_{\kappa}^{-}(t)$ and $\zeta_{\kappa}^{+}(t)=\zeta_{\kappa}^{-}(t)$, we may set $\zeta_{\kappa}^{v}=\zeta_{\kappa}^{+}=-\zeta_{\kappa}^{-}$and $\zeta_{\kappa}^{z}=\zeta_{\kappa}^{+}=\zeta_{\kappa}^{-}$. Then $(4.2)_{ \pm}$lead to the equations for $\zeta_{\kappa}^{v}(t)$ and $\zeta_{\kappa}^{z}(t)$ which are described, respectively, by

$$
\begin{aligned}
\frac{d \zeta_{\kappa}^{v}}{d t}= & -\varepsilon \kappa^{2} \zeta_{\kappa}^{v}-k\left[\chi^{\prime}\left(\sigma^{*}\right) \frac{e^{-2 \sqrt{\gamma} \eta^{*}}+e^{-2 \sqrt{\gamma+\kappa^{2}} \eta^{*}}}{2}-\chi^{\prime \prime}\left(\sigma^{*}\right) \frac{1-e^{-2 \sqrt{\gamma} \eta^{*}}}{2 \sqrt{\gamma}}\right. \\
& \left.\times \frac{\sqrt{\gamma+\kappa^{2}}-\sqrt{\gamma}-\sqrt{\gamma+\kappa^{2}} e^{-2 \sqrt{\gamma} \eta^{*}}-\sqrt{\gamma} e^{-2 \sqrt{\gamma+\kappa^{2}} \eta^{*}}}{2 \sqrt{\gamma} \sqrt{\gamma+\kappa^{2}}}\right] \zeta_{\kappa}^{v} \\
\equiv & A^{v}(\kappa) \zeta_{\kappa}^{v}
\end{aligned}
$$


and

$$
\begin{aligned}
\frac{d \zeta_{\kappa}^{z}}{d t}=- & \varepsilon \kappa^{2} \zeta_{\kappa}^{z}-k\left[\chi^{\prime}\left(\sigma^{*}\right) \frac{e^{-2 \sqrt{\gamma} \eta^{*}}-e^{-2 \sqrt{\gamma+\kappa^{2}} \eta^{*}}}{2}-\chi^{\prime \prime}\left(\sigma^{*}\right) \frac{1-e^{-2 \sqrt{\gamma} \eta^{*}}}{2 \sqrt{\gamma}}\right. \\
& \left.\times \frac{\sqrt{\gamma+\kappa^{2}}-\sqrt{\gamma}-\sqrt{\gamma+\kappa^{2}} e^{-2 \sqrt{\gamma} \eta^{*}}+\sqrt{\gamma} e^{-2 \sqrt{\gamma+\kappa^{2}} \eta^{*}}}{2 \sqrt{\gamma} \sqrt{\gamma+\kappa^{2}}}\right] \zeta_{\kappa}^{z} \\
\equiv & A^{z}(\kappa) \zeta_{\kappa}^{z} .
\end{aligned}
$$

Thus, one finds that the stability of the stationary interfaces $\Gamma_{ \pm}^{0}$ is determined by the signs of $A^{v}(\kappa)$ and $A^{z}(\kappa)$.

In the next section, we show that critical eigenvalues for the linearized problem (1.2)-(1.5) are given by the forms of $\varepsilon A^{v}(\kappa)+o(\varepsilon)$ and $\varepsilon A^{z}(\kappa)+o(\varepsilon)$ for sufficiently small $\varepsilon>0$.

\section{Stability of planar equilibrium solutions}

For sufficiently small $\varepsilon>0$, we consider the distribution of eigenvalues of the linearized problem of (1.2)-(1.5) around the planar equilibrium solution $\left(u^{\varepsilon}(x, y), v^{\varepsilon}(x, y)\right)$ in $\Omega_{\ell}$. The corresponding linearized eigenvalue problem is given by

$$
\begin{aligned}
& \lambda w=\varepsilon^{2} \Delta w-\varepsilon k\left[\chi^{\prime}\left(v^{\varepsilon}\right) v_{x}^{\varepsilon} w_{x}+\left(\chi^{\prime}\left(v^{\varepsilon}\right) v_{x}^{\varepsilon}\right)_{x} w\right]+f^{\prime}\left(u^{\varepsilon}\right) w-\varepsilon k\left[u^{\varepsilon} \chi^{\prime}\left(v^{\varepsilon}\right) \Delta z\right. \\
& \left.\quad+\left\{u_{x}^{\varepsilon} \chi^{\prime}\left(v^{\varepsilon}\right)+2 u^{\varepsilon} \chi^{\prime \prime}\left(v^{\varepsilon}\right) v_{x}^{\varepsilon}\right\} z_{x}+\left(u^{\varepsilon} \chi^{\prime \prime}\left(v^{\varepsilon}\right) v_{x}^{\varepsilon}\right)_{x} z\right], \quad(x, y) \in \Omega_{\ell}, \\
& \lambda z=w+\Delta z-\gamma z,
\end{aligned}
$$

with boundary conditions

$$
\begin{aligned}
& w(-\infty, y)=0=w(\infty, y) \\
& z(-\infty, y)=0=z(\infty, y)
\end{aligned}
$$

To solve that, we use the complete orthonormal system $\left\{Y_{m}\right\}_{m=0}^{\infty}$ in $L^{2}(0, \ell)$ where

$$
Y_{m}= \begin{cases}\ell^{-1 / 2} & \text { for } m=0 \\ \sqrt{2} \ell^{-1 / 2} \cos (m \pi y / \ell) & \text { for } m>0\end{cases}
$$

For $(w(x, y), z(x, y)) \in L^{2}\left(\Omega_{\ell}\right) \times L^{2}\left(\Omega_{\ell}\right)$, we define $\left(w_{m}(x), z_{m}(x)\right)$ by

$$
w_{m}(x)=\int_{0}^{\ell} w(x, y) Y_{m}(y) d y, \quad z_{m}(x)=\int_{0}^{\ell} z(x, y) Y_{m}(y) d y
$$

for $x \in \mathbf{R}, m=0,1,2, \ldots$ Then $(w(x, y), z(x, y))$ may be expanded to

$$
w(x, y)=\sum_{m=0}^{\infty} w_{m}(x) Y_{m}(y), \quad z(x, y)=\sum_{m=0}^{\infty} z_{m}(x) Y_{m}(y)
$$

in $L^{2}\left(\Omega_{\ell}\right)$. It follows from (5.1), (5.2) that for $\kappa=m \pi / \ell, w_{m}$ and $z_{m}$ satisfy

$$
\begin{aligned}
& \lambda w_{m}=\left(L^{\varepsilon}-\varepsilon^{2} \kappa^{2}\right) w_{m}-N^{\varepsilon, \kappa} z_{m}, \\
& \lambda z_{m}=w_{m}+\left(M-\kappa^{2}\right) z_{m}
\end{aligned}
$$


where

$$
\begin{aligned}
L^{\varepsilon} & \equiv \varepsilon^{2} \frac{d^{2}}{d x^{2}}-\varepsilon k \chi^{\prime}\left(v^{\varepsilon}\right) v_{x}^{\varepsilon} \frac{d}{d x}-\varepsilon k\left\{\chi^{\prime}\left(v^{\varepsilon}\right) v_{x x}^{\varepsilon}+\chi^{\prime \prime}\left(v^{\varepsilon}\right)\left(v_{x}^{\varepsilon}\right)^{2}\right\}+f^{\prime}\left(u^{\varepsilon}\right), \\
N^{\varepsilon, \kappa} & \equiv \varepsilon k\left[u^{\varepsilon} \chi^{\prime}\left(v^{\varepsilon}\right)\left\{\frac{d^{2}}{d x^{2}}-\kappa^{2}\right\}+\left\{u_{x}^{\varepsilon} \chi^{\prime}\left(v^{\varepsilon}\right)+2 u^{\varepsilon} \chi^{\prime \prime}\left(v^{\varepsilon}\right) v_{x}^{\varepsilon}\right\} \frac{d}{d x}+\left(u^{\varepsilon} \chi^{\prime \prime}\left(v^{\varepsilon}\right) v_{x}^{\varepsilon}\right)_{x}\right], \\
M & \equiv \frac{d^{2}}{d x^{2}}-\gamma .
\end{aligned}
$$

Substituting the second equation of $(5.3)_{m}$ into the first one, we rewrite $(5.3)_{m}$ as

$$
\begin{aligned}
& \left(T^{\varepsilon}-\varepsilon^{2} \kappa^{2}-\lambda\right) w_{m}=S^{\varepsilon}(\lambda) z_{m}, \\
& \left(M-\kappa^{2}-\lambda\right) z_{m}=-w_{m},
\end{aligned}
$$

where

$$
\begin{aligned}
T^{\varepsilon} & \equiv L^{\varepsilon}+\varepsilon k u^{\varepsilon} \chi^{\prime}\left(v^{\varepsilon}\right) \\
S^{\varepsilon}(\lambda) & \equiv \varepsilon k\left[\left\{u_{x}^{\varepsilon} \chi^{\prime}\left(v^{\varepsilon}\right)+2 u^{\varepsilon} \chi^{\prime \prime}\left(v^{\varepsilon}\right) v_{x}^{\varepsilon}\right\} \frac{d}{d x}+(\gamma+\lambda) u^{\varepsilon} \chi^{\prime}\left(v^{\varepsilon}\right)+\left(u^{\varepsilon} \chi^{\prime \prime}\left(v^{\varepsilon}\right) v_{x}^{\varepsilon}\right)_{x}\right] .
\end{aligned}
$$

The boundary conditions for $(5.4)_{m}$ are

$$
\begin{aligned}
& w_{m}(-\infty)=0=w_{m}(\infty) \\
& z_{m}(-\infty)=0=z_{m}(\infty)
\end{aligned}
$$

We note that to solve the eigenvalue problem $(5.3)_{m}$ with $(5.5)_{m}$ on $\mathbf{R}$ is equivalent to solving (5.4) $)_{m}$ on $\mathbf{R}_{+}$under the two types of boundary conditions

$$
\begin{aligned}
& w_{m}(\infty)=0=z_{m}(\infty), \\
& w_{m x}(0)=0=z_{m x}(0),
\end{aligned}
$$

and

$$
\begin{aligned}
& w_{m}(\infty)=0=z_{m}(\infty), \\
& w_{m}(0)=0=z_{m}(0) .
\end{aligned}
$$

Here, the boundary conditions $(5.6)_{N}$ and $(5.6)_{D}$ correspond to those for the linearized eigenvalue problem with varicose and zig-zag disturbances, respectively.

Remark 1. By differentiating (3.6) with respect to $x$ and using (3.7), one finds that $\left(u_{x}^{\varepsilon}(x), v_{x}^{\varepsilon}(x)\right)$ is the eigenfunction corresponding to the zero eigenvalue of $(5.4)_{0}$ with $(5.6)_{D}$.

For the distribution of non-zero eigenvalues, we first show

Proposition 1. There exists a positive constant $\theta$ such that for any given $\delta>0$, there is an $\varepsilon_{0}>0$ such that any eigenvalue $\lambda \in \mathbf{C}$ of $(5.4)_{m},(5.5)_{m}$ satisfies either $\operatorname{Re} \lambda<-\theta$ or

$$
\left|\varepsilon^{2} \kappa^{2}+\lambda\right|<\delta
$$

for any $0<\varepsilon<\varepsilon_{0}$ and $\kappa>0$.

The proof of this proposition is given in Section 7.

This proposition indicates that any eigenvalues not satisfying (5.7) are uniformly away from the imaginary axis in the left-half plane. On the other hand, the distribution of eigenvalues satisfying (5.7) is given as follows. 
Theorem 2. Let $m \in \mathbf{Z}$ and $\ell \in \mathbf{R}_{+}$be arbitrarily fixed. Then there are critical eigenvalues $\lambda_{N}^{m}$ and $\lambda_{D}^{m}$ of $(5.4)_{m}$ with $(5.6)_{N}$ and $(5.6)_{D}$ which are simple and satisfy

$$
\lim _{\varepsilon \downarrow 0}\left\{\frac{\lambda_{N}^{m}}{\varepsilon}-A^{v}\left(\frac{m \pi}{\ell}\right)\right\}=0 \quad \text { and } \quad \lim _{\varepsilon \downarrow 0}\left\{\frac{\lambda_{D}^{m}}{\varepsilon}-A^{z}\left(\frac{m \pi}{\ell}\right)\right\}=0
$$

where $A^{v}$ and $A^{z}$ are the quantities defined in (4.3) and (4.4).

The proof is given in Section 7. This theorem implies that the stability of the equilibrium solution is determined by the eigenvalues of the limiting system.

In the next section, we apply Theorem 2 to consider the stability of the planar equilibrium solution of (1.2)-(1.4) for some specific forms of $\chi(v)$.

\section{Applications}

For the stability of the 1-dimensional equilibrium solution, we know

Corollary 1. Let $\eta^{*}>0$ be defined by $H\left(\eta^{*}\right)=0$ (see (3.3)). The 1-dimensional symmetric equilibrium solution of (1.2)-(1.5) for small $\varepsilon>0$ is stable if $\frac{d}{d \eta} H\left(\eta^{*}\right)<0$, while it is unstable if $\frac{d}{d \eta} H\left(\eta^{*}\right)>0$.

Proof. Since the disturbance is only the case of $m=0$, that is, $\kappa=0$, it immediately follows from Theorem 2 that $A^{v}(0)=\frac{d}{d \eta} H\left(\eta^{*}\right)$ and $A^{z}(0)=0$. Therefore, the stability is determined by the sign of $\frac{d}{d \eta} H\left(\eta^{*}\right)$.

Remark 2. Corollary 1 and Theorem 2 say that the 1-dimensional equilibrium solutions corresponding to the branches of Figures 1, 2(i), and the lower one of Figure 2(ii) for small $\varepsilon>0$ are stable in $\mathbf{R}$, while the solutions to the upper branch of Figure 2(ii) are unstable.

Next, we consider the stability of the planar equilibrium solution. When the width of the strip domain is small, stability is determined by the 1-dimensional stability which is valid independently of the form of $\chi(v)$.

Corollary 2. There exists a positive constant $\omega$ such that when $\ell$ satisfies $0<\ell \leq$ $\pi \omega \varepsilon^{1 / 2}$, for sufficiently small $\varepsilon>0$, the planar equilibrium solution is stable (resp., unstable) if $\frac{d}{d \eta} H\left(\eta^{*}\right)<0$ (resp., $>0$ ).

Proof. If $\kappa>0$, that is, $m>0$, it follows from (4.3) and (4.4) that

$A^{v}\left(\frac{m \pi}{\ell}\right)=-\varepsilon \kappa^{2}+G+o(1)$ and $A^{z}\left(\frac{m \pi}{\ell}\right)=-\varepsilon \kappa^{2}+G+o(1)$ for large $\kappa>0$ where

$$
G=-\frac{k}{2}\left[\chi^{\prime}\left(\sigma^{*}\right) e^{-2 \sqrt{\gamma} \eta^{*}}-\frac{\chi^{\prime \prime}\left(\sigma^{*}\right)\left(1-e^{-2 \sqrt{\gamma} \eta^{*}}\right)^{2}}{2 \gamma}\right] .
$$

Therefore, there exists a positive function $\omega(\varepsilon)$ with $\omega(\varepsilon)=O(1)$ as $\varepsilon \rightarrow 0$ such that $\lambda_{N}^{m}<0$ and $\lambda_{D}^{m}<0(m>0)$ if $\omega^{-2} \leq \varepsilon \kappa^{2}$, that is, it is enough that $0<\ell<\pi \omega \varepsilon^{1 / 2}$ holds because of $\kappa=m \pi / \ell$. By Corollary 1 , this corollary is completely proved.

The case when the width $\ell$ is not so small is not simple. The stability depends on the form of $\chi(v)$. Fix $\ell$ arbitrarily. (i) With $\chi(v)=v$, the planar equilibrium solution is always stable for sufficiently small $\varepsilon>0$ because it follows from (4.3) and (4.4) that $A^{v}$ and $A^{z}$ are negative for $\ell>0$. (ii) With $\chi(v)=s v^{2} /\left(s+v^{2}\right)$, the stability of the planar equilibrium solution changes depending on the width $\ell>0$ as well as the other parameters $s>0$ and $k>0$. When $s$ is small, that is, the case of 
Figure 2(ii), the solution corresponding to the lower branch is stable for any $k>\underline{k}$ and $\ell>0$ for sufficiently small $\varepsilon>0$ where $\underline{k}$ is the turning point of the branch. But, for suitable large fixed $s>0$ and $\ell>0$, that is, the case of Figure 2(i), when $k$ decreases, the instability primarily appears with respect to zig-zag disturbances and secondarily appears with respect to varicose ones as in Figure 3 . We note that a similar bifurcation structure is demonstrated for a class of reaction-diffusion systems with activator-inhibitor interaction (Ohta, Mimura, and Kobayashi [21]).

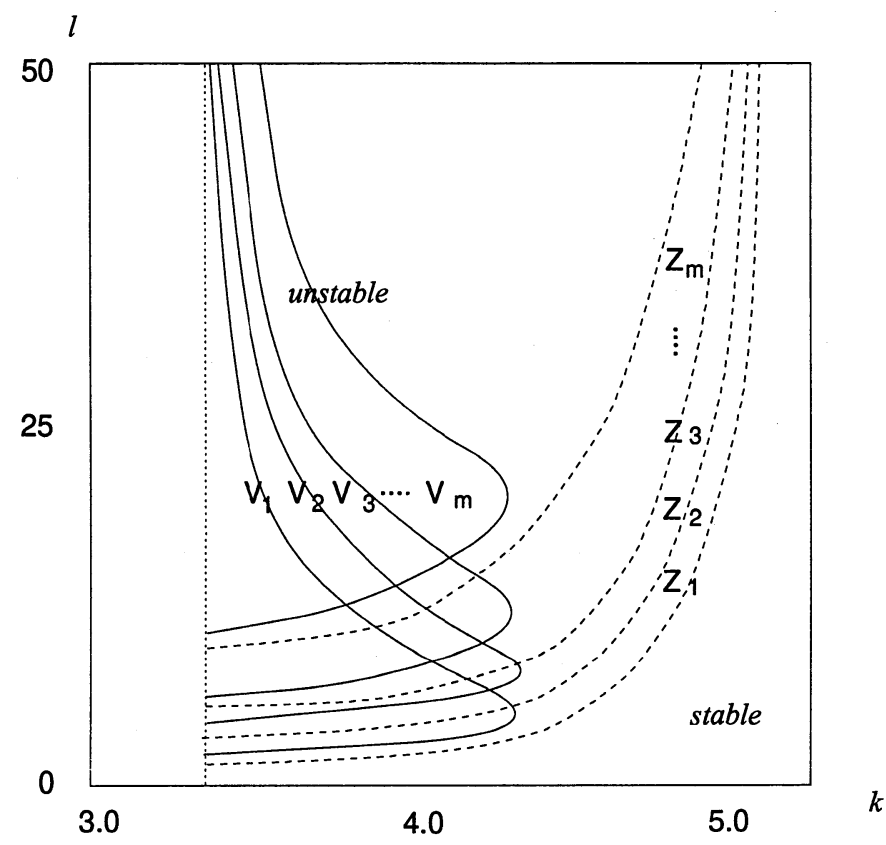

FIGURE 3. For the equilibrium solution corresponding to the branch in Figure 2 (i), at the lines $Z_{m}$ and $V_{m}$ the solution is neutrally stable with respect to $m$-mode zig-zag and varicose disturbances, respectively.

\section{Proofs}

Here we prove Theorem 1, Proposition 1, and Theorem 2.

Proof of Theorem 1. We first construct a solution of (3.6), (3.7) in two subintervals $(0, \eta)$ and $(\eta, \infty)$ with the following boundary condition at $x=\eta$ :

$$
(u(\eta), v(\eta))=(\zeta, \sigma)
$$

where $\zeta$ and $\sigma$ are arbitrary constants to be determined later. In order to do that, we construct an outer solution of $(3.6),(3.7)$ in $(0, \eta)$ and $(\eta, \infty)$ and an inner solution in a neighborhood of $x=\eta$ (see [7, 14], for instance).

Outer solutions. When $\varepsilon=0,(3.6)$ becomes

$$
\begin{aligned}
& 0=f(u), \\
& 0=v_{x x}+u-\gamma v, \quad x \in(0, \infty) .
\end{aligned}
$$


Solving the first equation in the above system, we take

$$
u_{0}(x)= \begin{cases}1 & x \in(0, \eta) \\ 0 & x \in(\eta, \infty)\end{cases}
$$

and put it into the second equation with the boundary conditions (3.7) and (7.1). Then the solution $v_{0}(x)$ is represented by (3.5). We call $\left(u_{0}(x), v_{0}(x)\right)$ the outer solution of (3.6), (3.7) in the limit $\varepsilon \downarrow 0$. It follows from (3.2) that

$$
\frac{d}{d x} v_{0}(\eta+0)=-\sigma \sqrt{\gamma} \quad \text { and } \quad \frac{d}{d x} v_{0}(\eta-0)=\left(\sigma-\frac{1}{\gamma}\right) \sqrt{\gamma} \frac{e^{2 \sqrt{\gamma} \eta}-1}{e^{2 \sqrt{\gamma} \eta}+1}
$$

We remark that when $\sigma=\sigma^{*}=\frac{1-e^{2 \sqrt{\gamma} \eta^{*}}}{2 \gamma}, v_{0}(x)$ belongs to $C^{1}(\mathbf{R})$.

Inner solutions. Since $v_{0}(x)$ is continuous in $\mathbf{R}_{+}$, but $u_{0}(x)$ is discontinuous at $x=\eta$, we look for an approximate solution to $(u(x), v(x))$ in a neighborhood of $x=\eta$. To do so, we use the usual stretched variable $\xi=(x-\eta) / \varepsilon$ so that (3.6) is rewritten as

$$
\begin{aligned}
& 0=u_{\xi \xi}-k u_{\xi} \chi^{\prime}(v) v_{x}-\varepsilon k u\left\{\chi^{\prime}(v) v_{x}\right\}_{x}+f(u) \\
& 0=v_{\xi \xi}+\varepsilon^{2}(u-\gamma v)
\end{aligned}
$$

We look for an approximate solution of (7.2) in the form

$$
\begin{aligned}
& u(\xi)=u_{0}(\varepsilon \xi+\eta)+U_{0}(\xi), \\
& v(\xi)=v_{0}(\varepsilon \xi+\eta)+V_{0}(\xi)+\varepsilon^{2} V_{2}(\xi)
\end{aligned}
$$

Substituting (7.3) into (7.2), we have

$$
\begin{aligned}
0=U_{0 \xi \xi}(\xi)-k\left\{U_{0 \xi}(\xi) \chi^{\prime}\left(v_{0}(\varepsilon \xi+\eta)+V_{0}(\xi)+\varepsilon^{2} V_{2}(\xi)\right)\right. & \\
& \times\left(v_{0 x}(\varepsilon \xi+\eta)+\frac{1}{\varepsilon} V_{0 \xi}(\xi)+\varepsilon V_{2 \xi}(\xi)\right)+\varepsilon\left(u_{0}(\varepsilon \xi+\eta)+U_{0}(\xi)\right) \\
& \times\left[\chi^{\prime}\left(v_{0}(\varepsilon \xi+\eta)+V_{0}(\xi)+\varepsilon^{2} V_{2}(\xi)\right)\left(v_{0 x x}(\varepsilon \xi+\eta)+\frac{1}{\varepsilon^{2}} V_{0 \xi \xi}(\xi)+V_{2 \xi \xi}(\xi)\right)\right. \\
& \left.\left.+\varepsilon \chi^{\prime \prime}\left(v_{0}(\varepsilon \xi+\eta)+V_{0}(\xi)+\varepsilon^{2} V_{2}(\xi)\right)\left(v_{0 x}(\varepsilon \xi+\eta)+\frac{1}{\varepsilon} V_{0 \xi}(\xi)+\varepsilon V_{2 \xi}(\xi)\right)^{2}\right]\right\} \\
& +f\left(u_{0}(\varepsilon \xi+\eta)+U_{0}(\xi)\right)+O(\varepsilon), \\
0= & v_{0 x x}(\varepsilon \xi+\eta)+\frac{1}{\varepsilon^{2}} V_{0 \xi \xi}(\xi)+V_{2 \xi \xi}(\xi)+u_{0}(\varepsilon \xi+\eta)+U_{0}(\xi) \\
& -\gamma\left(v_{0}(\varepsilon \xi+\eta)+V_{0}(\xi)+\varepsilon^{2} V_{2}(\xi)\right)+O(\varepsilon)
\end{aligned}
$$


for small $\varepsilon$. Noting that the interval $\mathbf{I}_{\varepsilon}$ becomes the whole interval $\mathbf{R}$ in the limit $\varepsilon \downarrow 0$, we impose the boundary conditions for $U_{0}, V_{0}$, and $V_{2}$,

$$
\begin{array}{ll}
\lim _{\xi \rightarrow-\infty} U_{0}(\xi)=0, & \lim _{\xi \rightarrow \infty} U_{0}(\xi)=0, \\
\lim _{\xi \rightarrow-0} U_{0}(\xi)=\zeta-1, & \lim _{\xi \rightarrow+0} U_{0}(\xi)=\zeta,
\end{array}
$$

and

$$
\begin{array}{ll}
\lim _{|\xi| \rightarrow \infty} V_{0}(\xi)=0, & \lim _{|\xi| \rightarrow \infty} V_{2}(\xi)=0, \\
V_{0}(0)=0, & V_{2}(0)=0 .
\end{array}
$$

When $\varepsilon$ tends to zero in (7.4), the second equation implies $V_{0 \xi \xi}=0$ so that $V_{0}(\xi) \equiv 0$ by (7.6), and then the first equation of (7.4) is

$$
0=U_{0 \xi \xi}(\xi)+\lambda U_{0 \xi}(\xi)+f\left(u_{0}(\eta)+U_{0}(\xi)\right)
$$

where $\lambda=-k \chi^{\prime}\left(v_{0}(\eta)\right) v_{0 x}(\eta)$. By putting

$$
W_{0}(\xi)= \begin{cases}U_{0}(\xi)+1, & \xi \in \mathbf{R}_{-}, \\ U_{0}(\xi), & \xi \in \mathbf{R}_{+},\end{cases}
$$

it follows from (7.5), (7.7) that $W_{0}$ satisfies

$$
\begin{gathered}
0=W_{0 \xi \xi}+\lambda W_{0 \xi}+f\left(W_{0}\right), \quad \xi \in \mathbf{R}_{ \pm}, \\
\lim _{\xi \rightarrow-\infty} W_{0}(\xi)=1, \quad \lim _{\xi \rightarrow \infty} W_{0}(\xi)=0, \quad W_{0}(0)=\zeta .
\end{gathered}
$$

Lemma 1. (i) There is $a \bar{c}>0$ such that when $\lambda=\bar{c}$, (7.8) has a unique monotone decreasing solution $W_{0}^{*}(\xi)$ in $\mathbf{R}$.

(ii) There exists $\delta>0$ such that for any $\lambda(|\lambda-\bar{c}|<\delta)$, (7.8) has a unique monotone solution $W_{0}^{ \pm}(\xi ; \lambda)$ in $\mathbf{R}_{ \pm}$, which satisfy $W_{0}^{ \pm}(\xi ; \bar{c})=W_{0}^{*}(\xi)$ in $\mathbf{R}_{ \pm}$and

$$
\left.\frac{\partial}{\partial \lambda}\left[W_{0 \xi}^{+}(0 ; \lambda)-W_{0 \xi}^{-}(0 ; \lambda)\right]\right|_{\lambda=\bar{c}}>0 .
$$

A proof is in Hosono and Mimura [11].

Thus, we have obtained $\left(U_{0}(\xi), V_{0}(\xi)\right)$. We next consider $V_{2}(\xi)$, which satisfies

$$
\begin{aligned}
& 0=V_{2 \xi \xi}+U_{0}(\xi), \quad \xi \in \mathbf{R}_{ \pm}, \\
& V_{2}( \pm \infty)=0, \quad V_{2}(0)=0 .
\end{aligned}
$$

Obviously the solution of (7.9) except for the boundary condition at $\xi=0$ is given by $\bar{V}_{2}^{ \pm}(\xi)=-\int_{\xi}^{ \pm \infty} \int_{s}^{ \pm \infty} U_{0}(t) d t d s$ in $\mathbf{R}_{ \pm}$. In order that $V_{2}(\xi)$ satisfy the boundary condition at $\xi=0$, we let

$$
V_{2}(\xi) \equiv \begin{cases}\bar{V}_{2}^{-}(\xi)-\bar{V}_{2}^{-}(0) e^{+\mu \xi}, & \xi \in \mathbf{R}_{-}, \\ \bar{V}_{2}^{+}(\xi)-\bar{V}_{2}^{+}(0) e^{-\mu \xi}, & \xi \in \mathbf{R}_{+},\end{cases}
$$

with some constant $\mu>0$.

Thus, we can construct an approximate solution $(u(\xi), v(\xi))$ of the form (7.3), which is called the inner solution of (3.6), (3.7), (7.1) with the stretched variable $\xi=(x-\eta) / \varepsilon$. 
We now construct the classical solution on $(0, \eta)$ by using the outer and inner solutions. By the new variable $y=x / \eta,(3.6)$ is rewritten as

$$
\begin{aligned}
& 0=\varepsilon^{2} u_{y y}-\varepsilon k u_{y} \chi^{\prime}(v) v_{y}-\varepsilon k u\left\{\chi^{\prime}(v) v_{y}\right\}_{y}+\eta^{2} f(u), \quad y \in(0,1) \\
& 0=v_{y y}+\eta^{2}(u-\gamma v)
\end{aligned}
$$

We look for a solution of (7.10) with (3.7), (7.1) in the form

$$
\begin{aligned}
& u(y ; \varepsilon, \eta, \sigma)=u_{0}(\eta y)+U_{0}(\eta(y-1) / \varepsilon) \theta(y)+p(y) \\
& v(y ; \varepsilon, \eta, \sigma)=v_{0}(\eta y)+\varepsilon^{2} V_{2}(\eta(y-1) / \varepsilon) \theta(y)+q(y)
\end{aligned}
$$

where $(p(y), q(y))$ is a remainder term with respect to small $\varepsilon$ and $\theta(y)$ is a smooth monotone function satisfying

$$
\theta(y) \equiv \begin{cases}0, & y \in\left(0, \frac{1}{2}\right) \\ 1, & y \in\left(\frac{3}{4}, 1\right)\end{cases}
$$

Substituting (7.11) into (7.10) with $t=(p, q)$, we have

$$
\begin{aligned}
P(t ; \varepsilon, \eta, \sigma) \equiv & \eta^{2} U_{0 \xi \xi} \theta+2 \varepsilon \eta U_{0 \xi} \theta_{y}+\varepsilon^{2} U_{0} \theta_{y y}+\varepsilon^{2} p_{y y} \\
- & \varepsilon k\left\{\left(\frac{\eta U_{0 \xi} \theta}{\varepsilon}+U_{0} \theta_{y}+p_{y}\right) \chi^{\prime}\left(v_{0}+\varepsilon^{2} V_{2} \theta+q\right)\right. \\
& \times\left(\eta v_{0 x}+\varepsilon \eta V_{2 \xi} \theta+\varepsilon^{2} V_{2} \theta_{y}+q_{y}\right) \\
+ & \left(u_{0}+U_{0} \theta+p\right)\left[\chi^{\prime \prime}\left(v_{0}+\varepsilon^{2} V_{2} \theta+q\right)\left(\eta v_{0 x}+\varepsilon \eta V_{2 \xi} \theta+\varepsilon^{2} V_{2} \theta_{y}+q_{y}\right)^{2}\right. \\
+ & \left.\left.\chi^{\prime}\left(v_{0}+\varepsilon^{2} V_{2} \theta+q\right)\left(\eta^{2} v_{0 x x}+\eta^{2} V_{2 \xi \xi} \theta+2 \varepsilon \eta V_{2 \xi} \theta_{y}+\varepsilon^{2} V_{2} \theta_{y y}+q_{y y}\right)\right]\right\} \\
+ & \eta^{2} f\left(u_{0}+U_{0} \theta+p\right), \\
Q(t ; \varepsilon, \eta, \sigma) \equiv & \eta^{2} v_{0 x x}+\eta^{2} V_{2 \xi \xi} \theta+2 \varepsilon \eta V_{2 \xi} \theta_{y}+\varepsilon^{2} V_{2} \theta_{y y}+q_{y y} \\
& +\eta^{2}\left\{u_{0}+U_{0} \theta+p-\gamma\left(v_{0}+\varepsilon^{2} V_{2} \theta+q\right)\right\} \\
= & 2 \varepsilon \eta V_{2 \xi} \theta_{y}+\varepsilon^{2} V_{2} \theta_{y y}+q_{y y}+\frac{n-1}{y}\left(\varepsilon \eta V_{2 \xi} \theta+\varepsilon^{2} V_{2} \theta_{y}+q_{y}\right) \\
& +\eta^{2}\left\{p-\gamma\left(\varepsilon^{2} V_{2} \theta+q\right)\right\}
\end{aligned}
$$

for $y \in(0,1)$. The boundary conditions are

$$
p_{y}(0)=q_{y}(0)=p(1)=q(1)=0
$$

We prove that there exists a solution $(p(y), q(y))$ by applying the usual implicit function theorem to $P(t ; \varepsilon, \eta, \sigma)=0$ and $Q(t ; \varepsilon, \eta, \sigma)=0$. We first note that the following 
six properties hold for small $\varepsilon$

$$
\begin{aligned}
P(0 ; \varepsilon, \eta, \sigma)= & 2 \varepsilon \eta U_{0 \xi} \theta_{y}+\varepsilon^{2} U_{0} \theta_{y y} \\
& -\varepsilon k\left(\frac{\eta}{\varepsilon} U_{0 \xi} \theta+U_{0} \theta_{y}\right) \chi^{\prime}\left(v_{0}+\varepsilon^{2} V_{2} \theta\right)\left(\eta v_{0 x}+\varepsilon \eta V_{2 \xi} \theta+\varepsilon^{2} V_{2} \theta_{y}\right) \\
& -\varepsilon k\left(u_{0}+U_{0} \theta\right)\left\{\chi^{\prime \prime}\left(v_{0}+\varepsilon^{2} V_{2} \theta\right)\left(\eta v_{0 x}+\varepsilon \eta V_{2 \xi} \theta+\varepsilon^{2} V_{2} \theta_{y}\right)^{2}\right. \\
+ & \chi^{\prime}\left(v_{0}+\varepsilon^{2} V_{2} \theta\right)\left(\eta^{2} v_{0 x x}+\eta^{2} V_{2 \xi \xi} \theta+2 \varepsilon \eta V_{2 \xi} \theta_{y}+\varepsilon^{2} V_{2} \theta_{y y}\right. \\
& \left.\left.+\varepsilon \eta V_{2 \xi} \theta+\varepsilon^{2} V_{2} \theta_{y}\right)\right\}+\eta^{2}\left\{f\left(u_{0}+U_{0} \theta\right)-f\left(u_{0}(0)+U_{0}\right)\right\} \\
& +k \eta^{2} \chi^{\prime}\left(v_{0}(\eta)\right) v_{0 x}(\eta) U_{0 \xi} \\
= & \eta^{2}\left\{-k\left[\chi^{\prime}\left(v_{0}\right) v_{0 x}-\chi^{\prime}\left(v_{0}(\eta)\right) v_{0 x}(\eta)\right] U_{0 \xi} \theta\right. \\
& \left.+f\left(u_{0}+U_{0} \theta\right)-f\left(u_{0}(0)+U_{0}\right)\right\}+O(\varepsilon) \\
= & O(\varepsilon), \\
Q(0 ; \varepsilon, \eta, \sigma)= & 2 \varepsilon \eta V_{2 \xi} \theta_{y}+\varepsilon^{2} V_{2} \theta_{y y}-\eta^{2} \gamma \varepsilon^{2} V_{2} \theta=O(\varepsilon),
\end{aligned}
$$

and

$$
\begin{aligned}
P_{p}(0 ; \varepsilon, \eta, \sigma)= & \varepsilon^{2} \frac{d^{2}}{d y^{2}}-\varepsilon k \chi^{\prime}\left(v_{0}+\varepsilon^{2} V_{2} \theta\right)\left(\eta v_{0 x}+\varepsilon \eta V_{2 \xi} \theta+\varepsilon^{2} V_{2} \theta_{y}\right) \frac{d}{d y} \\
& -\varepsilon k\left\{\chi^{\prime \prime}\left(v_{0}+\varepsilon^{2} V_{2} \theta\right)\left(\eta v_{0 x}+\varepsilon \eta V_{2 \xi} \theta+\varepsilon^{2} V_{2} \theta_{y}\right)^{2}\right. \\
& \left.+\chi^{\prime}\left(v_{0}+\varepsilon^{2} V_{2} \theta\right)\left(\eta^{2} v_{0 x x}+\eta^{2} V_{2 \xi \xi} \theta+2 \varepsilon \Gamma V_{2 \xi} \theta_{y}+\varepsilon^{2} V_{2} \theta_{y y}\right)\right\} \\
& +\eta^{2} f^{\prime}\left(u_{0}+U_{0} \theta\right) \\
= & \varepsilon^{2} \frac{d^{2}}{d y^{2}}-\varepsilon k \eta \chi^{\prime}\left(v_{0}\right) v_{0 x} \frac{d}{d y}+\eta^{2} f^{\prime}\left(u_{0}+U_{0} \theta\right)+O(\varepsilon), \\
P_{q}(0 ; \varepsilon, \eta, \sigma)=- & k\left\{\left(\eta U_{0 \xi} \theta+\varepsilon U_{0} \theta_{y}\right) \chi^{\prime \prime}\left(v_{0}+\varepsilon^{2} V_{2} \theta\right)\left(v_{0 x}+\varepsilon V_{2 \xi} \theta\right)+\chi^{\prime}\left(v_{0}+\varepsilon^{2} V_{2} \theta\right) \frac{d}{d y}\right. \\
& +\varepsilon\left(u_{0}+U_{0} \theta\right)\left[\chi^{\prime \prime \prime}\left(v_{0}+\varepsilon^{2} V_{2} \theta\right)\left(\eta v_{0 x}+\varepsilon \eta V_{2 \xi} \theta+\varepsilon^{2} V_{2} \theta_{y}\right)^{2}\right. \\
& +\chi^{\prime \prime}\left(v_{0}+\varepsilon^{2} V_{2} \theta\right) 2 \varepsilon\left(\eta v_{0 x}+\varepsilon \eta V_{2 \xi} \theta+\varepsilon^{2} V_{2} \theta_{y}\right) \frac{d}{d y} \\
& +\chi^{\prime \prime}\left(v_{0}+\varepsilon^{2} V_{2} \theta\right)\left(\eta^{2} v_{0 x x}+\eta^{2} V_{2 \xi \xi} \theta+2 \varepsilon \eta V_{2 \xi} \theta_{y}+\varepsilon^{2} V_{2} \theta_{y y}\right) \\
& \left.\left.+\chi^{\prime}\left(v_{0}+\varepsilon^{2} V_{2} \theta\right) \frac{d^{2}}{d y^{2}}\right]\right\} \\
Q_{p}(0 ; \varepsilon, \eta, \sigma)= & \eta^{2}, \\
Q_{q}(0 ; \varepsilon, \eta, \sigma)= & \frac{d^{2}}{d y^{2}}-\eta_{0 \xi} \theta\left\{\eta \chi^{\prime \prime}\left(v_{0}\right) v_{0 x}+\chi^{\prime}\left(v_{0}\right) \frac{d}{d y}\right\}+O(\varepsilon), \\
\left.Q_{p}\right) &
\end{aligned}
$$

where $P_{p}, P_{q}, Q_{p}$, and $Q_{q}$ mean the derivatives of $P$ and $Q$ with respect to $p$ and $q$. We note the following: (i) $Q_{q}(0 ; \varepsilon, \eta, \sigma)$ is invertible in a suitable function space with (7.13); (ii) $P_{q}(0 ; \varepsilon, \eta, \sigma)=O\left(\varepsilon^{\frac{1}{2}}\right)$ holds for small $\varepsilon$ with respect to the $L^{1}-$ norm; and (iii) since $f^{\prime}\left(u_{0}+U_{0}\right)<0$ in $(0,1)$ for any fixed $\zeta$ close to $1, P_{p}$ is invertible by the maximum principle of elliptic equations. Then, it turns out that the linearized operator $\left(\begin{array}{ll}P_{p} & P_{q} \\ Q_{p} & Q_{q}\end{array}\right)$ at $(p, q)=(0,0)$ is invertible for small $\varepsilon$ and $k$. It follows from 
the implicit function theorem in [7] that there is a solution $(p(y ; \varepsilon, \eta, \sigma), q(y ; \varepsilon, \eta, \sigma))$ of $P=Q=0$. Therefore, we have a solution $(u(y ; \varepsilon, \eta, \sigma), v(y ; \varepsilon, \eta, \sigma))$ of $(3.6),(3.7)$, (7.1) on $(0, \eta)$ of the form (7.11).

Next, we construct a solution of $(3.6),(3.7),(7.1)$ in $(\eta, \infty)$. By using the new variable $\rho=x-\eta,(3.6)$ is rewritten as

$$
\begin{aligned}
& 0=\varepsilon^{2} u_{\rho \rho}-\varepsilon k u_{\rho} \chi^{\prime}(v) v_{\rho}-\varepsilon k u\left\{\chi^{\prime}(v) v_{\rho}\right\}_{\rho}+f(u), \quad \rho \in \mathbf{R}_{+} \\
& 0=v_{\rho \rho}+u-\gamma v
\end{aligned}
$$

The boundary conditions are

$$
(u(0), v(0))=(\zeta, \sigma) \quad \text { and } \quad \lim _{\rho \rightarrow \infty}(u(\rho), v(\rho))=(0,0) .
$$

We look for a solution of (7.12), (7.13) on $\mathbf{R}_{+}$in the form

$$
\begin{aligned}
& u(\rho ; \varepsilon, \eta, \sigma)=u_{0}(\rho+\eta)+U_{0}(\eta / \varepsilon)+p(\eta) \\
& v(\rho ; \varepsilon, \eta, \sigma)=v_{0}(\rho+\eta)+\varepsilon^{2} V_{2}(\rho / \varepsilon)+q(\rho)
\end{aligned}
$$

where $(p(\rho), q(\rho))$ is a remainder term with respect to small $\varepsilon$. Substituting (7.14) with $t=(p, q)$ into $(7.12),(7.13)$, we have

$$
\begin{aligned}
P(t ; \varepsilon, \eta, \sigma) \equiv & U_{0 \xi \xi}+\varepsilon^{2} p_{\eta \eta}-k \varepsilon\left(\frac{U_{0 \xi}}{\varepsilon}+p_{\rho}\right) \chi^{\prime}\left(v_{0}+\varepsilon^{2} V_{2}+q\right)\left(v_{0 r}+\varepsilon V_{2 \xi}+q_{\rho}\right) \\
& -k \varepsilon\left(u_{0}+U_{0}+p\right)\left\{\chi^{\prime \prime}\left(v_{0}+\varepsilon^{2} V_{2}+q\right)\left(v_{0 x}+\varepsilon V_{2 \xi}+q_{\rho}\right)^{2}\right. \\
& \left.+\chi^{\prime}\left(v_{0}+\varepsilon^{2} V_{2}+q\right)\left(v_{0 x x}+V_{2 \xi \xi}+q_{\rho \rho}\right)\right\}+f\left(u_{0}+U_{0}+p\right), \\
Q(t ; \varepsilon, \eta, \sigma) \equiv & v_{0 x x}+V_{2 \xi \xi}+q_{\rho \rho}+u_{0}+U_{0}+p-\gamma\left(v_{0}+\varepsilon^{2} V_{2}+q\right) \\
= & q_{\rho \rho}+p-\gamma\left(\varepsilon^{2} V_{2}+q\right) .
\end{aligned}
$$

The boundary conditions are given by

$$
p(0)=q(0)=p( \pm \infty)=q( \pm \infty)=0
$$

Therefore, we will prove that $P(t ; \varepsilon, \eta, \sigma)=0$ and $Q(t ; \varepsilon, \eta, \sigma)=0$ has a solution $(p(\rho), q(\rho))$ by using the implicit function theorem, as usual. To do that, we show the following six properties with respect to $P$ and $Q$ for small $\varepsilon$ :

$$
\begin{aligned}
& P(0 ; \varepsilon, \eta, \sigma)=- k U_{0 \xi} \chi^{\prime}\left(v_{0}+\varepsilon^{2} V_{2}\right)\left(v_{0 x}+\varepsilon V_{2 \xi}\right) \\
&-\varepsilon k\left(u_{0}+U_{0}\right)\left\{\chi^{\prime \prime}\left(v_{0}+\varepsilon^{2} V_{2}\right)\left(v_{0 x}+\varepsilon V_{2 \xi}\right)^{2}\right. \\
&\left.+\chi^{\prime}\left(v_{0}+\varepsilon^{2} V_{2}\right)\left(v_{0 x x}+V_{2 \xi \xi}\right)\right\}+k \chi^{\prime}\left(v_{0}(0)\right) v_{0 x}(0) U_{0 \xi} \\
&=- k\left\{\chi^{\prime}\left(v_{0}\right) v_{0 x}-\chi^{\prime}\left(v_{0}(0)\right) v_{0 x}(0)\right\} U_{0 \xi}+O(\varepsilon) \\
&= O(\varepsilon) \\
& Q(0 ; \varepsilon, \eta, \sigma)=-\gamma \varepsilon^{2} V_{2}=O(\varepsilon)
\end{aligned}
$$


and

$$
\begin{aligned}
P_{p}(0 ; \varepsilon, \eta, \sigma)= & \varepsilon^{2} \frac{d^{2}}{d \rho^{2}}-\varepsilon k \chi^{\prime}\left(v_{0}+\varepsilon^{2} V_{2}\right)\left(v_{0 x}+\varepsilon V_{2 \xi}\right) \frac{d}{d \rho} \\
& -\varepsilon k\left\{\chi^{\prime \prime}\left(v_{0}+\varepsilon^{2} V_{2}\right)\left(v_{0 x}+\varepsilon V_{2 \xi}\right)^{2}\right. \\
& \left.+\chi^{\prime}\left(v_{0}+\varepsilon^{2} V_{2}\right)\left(v_{0 x x}+V_{2 \xi \xi}\right)\right\}+f^{\prime}\left(u_{0}+U_{0}\right) \\
= & \varepsilon^{2} \frac{d^{2}}{d \rho^{2}}-\varepsilon k \chi^{\prime}\left(v_{0}\right) v_{0 x} \frac{d}{d \rho}+f^{\prime}\left(u_{0}+U_{0}\right)+O(\varepsilon), \\
P_{q}(0 ; \varepsilon, \eta, \sigma)=- & k \chi^{\prime \prime}\left(v_{0}+\varepsilon^{2} V_{2}\right)\left(v_{0 x}+\varepsilon V_{2 \xi}\right) U_{0 \xi}+\chi^{\prime}\left(v_{0}+\varepsilon^{2} V_{2}\right) U_{0 \xi} \frac{d}{d \rho} \\
& -\varepsilon k\left(u_{0}+U_{0}\right)\left\{\chi^{\prime \prime \prime}\left(v_{0}+\varepsilon^{2} V_{2}\right)\left(v_{0 x}+\varepsilon V_{2 \xi}\right)^{2}+\chi^{\prime \prime}\left(v_{0}+\varepsilon^{2} V_{2}\right)\right. \\
& \times 2\left(v_{0 x}+\varepsilon V_{2 \xi}\right) \frac{d}{d \rho}+\chi^{\prime \prime}\left(v_{0}+\varepsilon^{2} V_{2}\right)\left(v_{0 x x}+V_{2 \xi \xi}\right) \\
& \left.+\chi^{\prime}\left(v_{0}+\varepsilon^{2} V_{2}\right) \frac{d^{2}}{d \rho^{2}}\right\} \\
= & -k U_{0 \xi}\left\{\chi^{\prime \prime}\left(v_{0}\right) v_{0 x}+\chi^{\prime}\left(v_{0}\right) \frac{d}{d \eta}\right\}+O(\varepsilon), \\
Q_{p}(0 ; \varepsilon, \eta, \sigma)= & 1, \quad \\
Q_{q}(0 ; \varepsilon, \eta, \sigma)= & \frac{d^{2}}{d \rho^{2}}-\gamma .
\end{aligned}
$$

We note that $P_{q}(0 ; \varepsilon, \eta, \sigma)=O\left(\varepsilon^{\frac{1}{2}}\right)$ for small $\varepsilon$ with respect to the $L^{1}$-norm, and $Q_{q}(0 ; \varepsilon, \eta, \sigma)$ is invertible. In a way similar to the proof of Lemma 4 in Hosono and Mimura [11], we can show that $P_{p}$ is invertible. Thus the linearized operator of $(P, Q)$ at $(p, q)=(0,0)$ is invertible, so that the implicit function theorem implies that there exists a solution $(p(\rho ; \varepsilon, \eta, \sigma), q(\rho ; \varepsilon, \eta, \sigma))$ of $P=Q=0$. Therefore, (7.12), (7.13) has a solution of the form (7.14) on the half line $\mathbf{R}_{+}$.

Note that the solutions of the forms (7.11) and (7.14) for (3.6), (3.7), and (7.1) are continuous but not continuously differentiable at $x=\eta$. So, we show the existence of functions $\eta(\varepsilon)$ and $\sigma(\varepsilon)$ for small $\varepsilon$ such that each solution on $(0, \eta)$ and $(\eta, \infty)$ is continuously differentiable at $x=\eta$. By the form of $v_{0}(x),(7.11),(7.14),(3.4)$, and (ii) in Lemma 1, we find that at $\sigma=\sigma^{*}, \eta=\eta^{*}$, and $\lambda=c$,

$$
\begin{gathered}
\lim _{\varepsilon \downarrow 0} \frac{d}{d x}[v(\eta+0)-v(\eta-0)]=0, \\
\lim _{\varepsilon \downarrow 0} \frac{\partial}{\partial \sigma}\left[v_{x}(\eta+0)-v_{x}(\eta-0)\right]=\frac{-2 \gamma e^{2 \sqrt{\gamma} \eta}}{\sqrt{\gamma}\left(e^{2 \sqrt{\gamma} \eta}+1\right)}<0,
\end{gathered}
$$

and

$$
\begin{gathered}
\lim _{\varepsilon \downarrow 0} \varepsilon \frac{d}{d x}[u(\eta+0)-u(\eta-0)]=W_{0 \xi}(0+0)-W_{0 \xi}(0-0)=0 \\
\lim _{\varepsilon \downarrow 0} \frac{\partial}{\partial \eta}\left\{\varepsilon\left[u_{x}(\eta+0)-u_{x}(\eta-0)\right]\right\} \\
=k \frac{\partial}{\partial \eta}\left[\chi^{\prime}\left(v_{0}(\eta)\right) v_{0 x}(\eta)\right] \frac{\partial}{\partial \lambda}\left[W_{0 \xi}^{+}(0 ; \lambda)-W_{0 \xi}^{-}(0 ; \lambda)\right] \\
=\frac{\partial}{\partial \eta} H\left(\eta^{*}\right) \times \frac{1}{W_{0 \xi}^{*}(0)} \int_{-\infty}^{\infty} e^{c \xi}\left\{W_{0 \xi}^{*}(\xi)\right\}^{2} d \xi \neq 0 .
\end{gathered}
$$


By using the implicit function theorem, it follows that there exist $\sigma(\varepsilon)$ and $\eta(\varepsilon)$ such that for small $\varepsilon$, a solution $\left(u^{\varepsilon}(x), v^{\varepsilon}(x)\right)$ of the forms (7.11), (7.14) constructed on each interval becomes a solution of (3.6), (3.7) on $\mathbf{R}_{+}$. Therefore, the proof of Theorem 1 is completed.

Next, we prove Proposition 1 and Theorem 2. To do that, we first consider the following two eigenvalue problems:

$$
\begin{array}{ll}
T^{\varepsilon} \phi=\zeta \phi & \text { in } \mathbf{R}_{+} \\
\phi_{x}(0)=0, & \lim _{x \rightarrow \infty} \phi(x)=0
\end{array}
$$

and

$$
\begin{array}{ll}
T^{\varepsilon} \phi=\zeta \phi & \text { in } \mathbf{R}_{+}, \\
\phi(0)=0, & \lim _{x \rightarrow \infty} \phi(x)=0 .
\end{array}
$$

For each problem, the following lemma holds.

Lemma 2. Let $\sigma_{e}\left(T^{\varepsilon}\right)$ be the essential spectrum of $T^{\varepsilon}$. Then $\sigma_{e}\left(T^{\varepsilon}\right) \subset\left(-\infty, f^{\prime}(0)\right]$ holds, and all eigenvalues of $T^{\varepsilon}$ are real and simple.

The proof of this lemma is in Coddington and Levinson [5] and Henry [10], for instance.

Let $\left\{\zeta_{n}^{\varepsilon}\right\}_{n \geq 0},\left\{\zeta_{D, n}^{\varepsilon}\right\}_{n \geq 0}$ be the eigenvalues of the problems $(7.16)_{N},(7.16)_{D}$ and $\left\{\phi_{n}^{\varepsilon}\right\}_{n \geq 0},\left\{\phi_{D, n}^{\varepsilon}\right\}_{n \geq 0}$ be the corresponding orthonormal eigenfunctions, respectively.

By using the stretched variable $\xi=(x-\eta(\varepsilon)) / \varepsilon, \quad T^{\varepsilon}$ is rewritten as

$$
\tilde{T}^{\varepsilon}=\frac{d^{2}}{d \xi^{2}}-k \chi^{\prime}\left(\tilde{v}^{\varepsilon}\right) \tilde{v}_{x}^{\varepsilon} \frac{d}{d \xi}-\varepsilon k\left\{\chi^{\prime}\left(\tilde{v}^{\varepsilon}\right) \tilde{v}_{x x}^{\varepsilon}+\chi^{\prime \prime}\left(\tilde{v}^{\varepsilon}\right)\left(\tilde{v}_{x}^{\varepsilon}\right)^{2}-\tilde{u}^{\varepsilon} \chi^{\prime}\left(\tilde{v}^{\varepsilon}\right)\right\}+f^{\prime}\left(\tilde{u}^{\varepsilon}\right)
$$

in $[-\eta(\varepsilon) / \varepsilon, \infty)$, where $\tilde{u}^{\varepsilon}(\xi)=u^{\varepsilon}(\eta(\varepsilon)+\varepsilon \xi)$ and $\tilde{v}^{\varepsilon}(\xi)=v^{\varepsilon}(\eta(\varepsilon)+\varepsilon \xi)$. Note that

$$
\lim _{\varepsilon \downarrow 0} \tilde{T}^{\varepsilon} \equiv \tilde{T}^{0}=\frac{d^{2}}{d \xi^{2}}-k \chi^{\prime}\left(\sigma^{*}\right) v_{x}^{0}\left(\eta^{*}\right) \frac{d}{d \xi}+f^{\prime}\left(W_{0}\right) \quad \text { in } \mathbf{R}
$$

where $\lim _{\varepsilon \downarrow 0} \eta(\varepsilon)=\eta^{*}$ and $\lim _{\varepsilon \downarrow 0} \tilde{v}_{x}^{\varepsilon}(\xi)=v_{x}^{0}\left(\eta^{*}\right)$. Putting $\hat{\phi}_{n}^{\varepsilon}(\xi)=\sqrt{\varepsilon} \phi_{n}^{\varepsilon}(\eta(\varepsilon)+\varepsilon \xi)$, we find that $\hat{\phi}_{n}^{\varepsilon}$ is the eigenfunction corresponding to the eigenvalue $\zeta_{n}^{\varepsilon}$ of $\tilde{T}^{\varepsilon}$ with the Neumann boundary conditions.

Remark 3. $-\frac{d}{d \xi} W_{0}$ is a constant multiple of the positive normalized eigenfunction $\hat{\phi}_{0}^{*}$ corresponding to the principal eigenvalue 0 of the Sturm-Liouville operator $\tilde{T}^{0}$, and

$$
-\frac{d}{d \xi} \tilde{u}^{\varepsilon} \rightarrow \kappa^{*-1} \hat{\phi}_{0}^{*} \quad\left(=-\frac{d}{d \xi} W_{0}\right) \quad \text { as } \varepsilon \rightarrow 0
$$

where $\kappa^{*-1}=\left\|\frac{d}{d \xi} W_{0}\right\|_{L^{2}(\mathbf{R})}$. Moreover, the eigenvalues of $\tilde{T}^{0}$ are all simple and the remaining spectrum, except for the zero eigenvalue, is in the parabola $\{\lambda \in \mathbf{C} \mid-\operatorname{Re} \lambda \geq$ $\left.a(\operatorname{Im} \lambda)^{2}+b\right\}$ for some positive constants $a, b$ (see Sattinger [23], for instance).

Let $\phi_{0}^{\varepsilon *}$ be the eigenfunction corresponding to the principal eigenvalue $\zeta_{0}^{\varepsilon *}$ of the adjoint operator $T^{\varepsilon *}$ of $T^{\varepsilon}$, which is represented by

$$
T^{\varepsilon *}=\varepsilon^{2} \frac{d^{2}}{d x^{2}}+\varepsilon k \chi^{\prime}\left(v^{\varepsilon}\right) v_{x}^{\varepsilon} \frac{d}{d x}+\varepsilon k u^{\varepsilon} \chi^{\prime}\left(v^{\varepsilon}\right)+f^{\prime}\left(u^{\varepsilon}\right) .
$$

Lemma 3. (Nishiura and Fujii [19, Lemma 1.3]) It holds that

$$
\hat{\phi}_{0}^{\varepsilon} \quad\left(=\sqrt{\varepsilon} \tilde{\phi}_{0}^{\varepsilon}\right) \rightarrow \hat{\phi}_{0}^{*} \quad \text { in the } C_{c . u}^{2}(\mathbf{R}) \text {-sense } \quad \text { as } \varepsilon \downarrow 0
$$


and

$$
\hat{\phi}_{0}^{\varepsilon *} \rightarrow \gamma^{*} e^{c \xi} \hat{\phi}_{0}^{*} \quad \text { in the } C_{c . u}^{2}(\mathbf{R}) \text {-sense } \quad \text { as } \varepsilon \downarrow 0
$$

where $\gamma^{*}=1 /\left\|e^{c \xi / 2} \hat{\phi}_{0}^{*}\right\|_{L^{2}}^{2}$.

Corollary 3. [19, Corollary 1.3]

$$
\int_{0}^{\infty} \phi_{0}^{\varepsilon}(x) d x=L(\varepsilon) \sqrt{\varepsilon} \quad \text { and } \quad \int_{0}^{\infty} \phi_{0}^{\varepsilon *}(x) d x=L^{*}(\varepsilon) \sqrt{\varepsilon}
$$

where $L(\varepsilon)=\int_{-\eta(\varepsilon) / \varepsilon}^{\infty} \sqrt{\varepsilon} \phi_{0}^{\varepsilon}(\eta(\varepsilon)+\varepsilon \xi) d \xi$ and $L^{*}(\varepsilon)=\int_{-\eta(\varepsilon) / \varepsilon}^{\infty} \sqrt{\varepsilon} \phi_{0}^{\varepsilon *}(\eta(\varepsilon)+\varepsilon \xi) d \xi$. Moreover, $L(0)=\kappa^{*}$ and $L^{*}(0)=-\gamma^{*} \kappa^{*} \int_{-\infty}^{\infty} e^{c \xi} W_{0 \xi} d \xi$.

Lemma 4.

$$
\left\{\chi^{\prime}\left(\sigma^{*}\right)+\sigma^{*} \chi^{\prime \prime}\left(\sigma^{*}\right)\right\} \int_{-\infty}^{\infty}\left(W_{0 \xi}\right)^{2} e^{c \xi} d \xi-\chi^{\prime}\left(\sigma^{*}\right) \frac{2 e^{2 \sqrt{\gamma} \eta^{*}}}{1+e^{2 \sqrt{\gamma} \eta^{*}}} \int_{-\infty}^{0}\left(W_{0 \xi}\right)^{2} e^{c \xi} d \xi \neq 0
$$

where $W_{0}$ is the traveling front solution of (2.4).

Proof. Put $W_{0}(0)=\zeta$. Thus $\int_{-\infty}^{0}\left(W_{0 \xi}\right)^{2} e^{c \xi} d \xi \rightarrow 0$ as $\zeta \rightarrow 1$, so the proof of this lemma follows from (3.4).

Using Lemma 4, we have the two lemmas:

Lemma 5. [19, Lemma 1.4] Let $\zeta_{0}^{\varepsilon}$ be the principal eigenvalue of $T^{\varepsilon}$ with the Neumann or Dirichlet boundary condition. Then, we have $\zeta_{0}^{\varepsilon}=\varepsilon \hat{\zeta}_{0}(\varepsilon)+O\left(\varepsilon^{\frac{5}{2}}\right)$ for small $p$ and

$$
\begin{aligned}
\lim _{\varepsilon \downarrow 0} \hat{\zeta}_{0}(\varepsilon)=\hat{\zeta}_{0}^{*} & =k\left[\chi^{\prime}\left(\sigma^{*}\right) v_{x x}^{*}+\chi^{\prime \prime}\left(\sigma^{*}\right)\left(v_{x}^{*}\right)^{2}-\chi^{\prime}\left(\sigma^{*}\right) \frac{\left\langle\left(W_{0 \xi}\right)^{2}, e^{c \xi}\right\rangle_{\mathbf{R}_{-}}}{\left\langle\left(W_{0 \xi}\right)^{2}, e^{c \xi}\right\rangle_{\mathbf{R}}}\right] \\
& =k\left[\gamma \sigma^{*}\left\{\chi^{\prime}\left(\sigma^{*}\right)+\sigma^{*} \chi^{\prime \prime}\left(\sigma^{*}\right)\right\}-\chi^{\prime}\left(\sigma^{*}\right) \frac{\left\langle\left(W_{0 \xi}\right)^{2}, e^{c \xi}\right\rangle_{\mathbf{R}_{-}}}{\left\langle\left(W_{0 \xi}\right)^{2}, e^{c \xi}\right\rangle_{\mathbf{R}}}\right] \neq 0
\end{aligned}
$$

where $v_{x}^{*}$ and $v_{x x}^{*}$ are the values of $v_{x}^{0}$ and $v_{x x}^{0}$ at $x=\eta^{*}$ and $\langle\cdot, \cdot\rangle_{\mathbf{R}}$ and $\langle\cdot, \cdot\rangle_{\mathbf{R}_{ \pm}}$mean, respectively, the $L^{2}(\mathbf{R})$ - and $L^{2}\left(\mathbf{R}_{ \pm}\right)$-inner products. Moreover, there is a positive constant $\mu$ independent of $\varepsilon$ such that other eigenvalues $\zeta_{n}^{\varepsilon}$ of $T^{\varepsilon}$ satisfy $\zeta_{n}^{\varepsilon}<-\mu$ for any integer $n>0$.

Lemma 6. The principal eigenvalue $\zeta_{0}^{\varepsilon}$ of $T^{\varepsilon}$ does not belong to the spectrum of $(5.4)_{0}$, (5.6) for small $\varepsilon>0$.

This lemma will be proved at the end of this section.

Let $\Lambda_{1} \equiv\{\lambda \in \mathbf{C} \mid \operatorname{Re} \lambda>-\min \{\mu, \gamma, b\}\}$. We note that the constant $\theta>0$ in Proposition 1 is determined by $\theta=\min \{\mu, \gamma, b\}$. For $\lambda \in \Lambda_{1}$, we decompose $w_{m}$ into two parts as follows:

$$
\begin{aligned}
w_{m} & =\left(T^{\varepsilon}-\varepsilon^{2} \kappa^{2}-\lambda\right)^{-1} S^{\varepsilon}(\lambda) z_{m} \\
& \equiv \frac{\left\langle S^{\varepsilon}(\lambda) z_{m}, \phi_{0}^{\varepsilon *}\right\rangle_{\mathbf{R}_{+}}}{\zeta_{0}^{\varepsilon}-\varepsilon^{2} \kappa^{2}-\lambda} \phi_{0}^{\varepsilon}+\left(T^{\varepsilon}-\varepsilon^{2} \kappa^{2}-\lambda\right)^{\dagger} S^{\varepsilon}(\lambda) z_{m} .
\end{aligned}
$$

Here the first term in the right-hand side means the projection of the space spanned by the eigenfunction corresponding to the principal eigenvalue $\zeta_{0}^{\varepsilon}$ of $T^{\varepsilon}$. By using Lemma 6 , it is easy to show that $\left(T^{\varepsilon}-\lambda\right)^{\dagger}$ is a uniformly $L_{\rho}^{2}$ - bounded family of operators for $\lambda \in \Lambda_{1}$; more precisely, we have

$$
\left\|\left(T^{\varepsilon}-\lambda\right)^{\dagger}\right\|_{L_{\rho}^{2} \rightarrow L_{\rho}^{2}} \leq \frac{1}{\left|\zeta_{1}^{\varepsilon}-\lambda\right|}
$$


for some $\rho>0$. By the argument in the proof of Lemma 6 , we have $\zeta_{0}^{\varepsilon}-\varepsilon^{2} \kappa^{2}-\lambda \neq 0$. Substituting (7.17) into the second equation of $(5.4)_{m}$, we have

$$
\lambda z_{m}=z_{m x x}+\frac{\left\langle S^{\varepsilon}(\lambda) z_{m}, \phi_{0}^{\varepsilon *}\right\rangle_{\mathbf{R}_{+}}}{\zeta_{0}^{\varepsilon}-\varepsilon^{2} \kappa^{2}-\lambda} \phi_{0}^{\varepsilon}+\left(T^{\varepsilon}-\varepsilon^{2} \kappa^{2}-\lambda\right)^{\dagger} S^{\varepsilon}(\lambda) z_{m}-\left(\kappa^{2}+\gamma\right) z_{m} .
$$

Proof of Proposition 1. We prove this proposition by using the proof of Nishiura and Fujii [19]. Since the proof of Proposition 1 for the Dirichlet boundary conditions $(5.6)_{D}$ is the same as that for the Neumann boundary conditions $(5.6)_{N}$, we only consider the problem (7.19) with $(5.6)_{N}$. In order to do that, we introduce the following bilinear form associated with (7.19):

$$
\begin{aligned}
0=- & \left\langle z_{m x}, \psi_{x}\right\rangle_{\mathbf{R}_{+}}+\frac{\left\langle S^{\varepsilon}(\lambda) z_{m}, \phi_{0}^{\varepsilon *}\right\rangle_{\mathbf{R}_{+}}}{\zeta_{0}^{\varepsilon}-\varepsilon^{2} \kappa^{2}-\lambda}\left\langle\phi_{0}^{\varepsilon}, \psi\right\rangle_{\mathbf{R}_{+}} \\
& +\left\langle\left(T^{\varepsilon}-\varepsilon^{2} \kappa^{2}-\lambda\right)^{\dagger} S^{\varepsilon}(\lambda) z_{m}, \psi\right\rangle_{\mathbf{R}_{+}}-\left(\gamma+\kappa^{2}+\lambda\right)<z_{m}, \psi>_{\mathbf{R}_{+}}
\end{aligned}
$$

for $z_{m}, \psi \in H_{N}^{1}\left(\mathbf{R}_{+}\right)$where $z_{m}$ is normalized as $\left\|z_{m}\right\|_{L^{2}}=1$.

Lemma 7. There is a positive constant $M_{c}$ independent of $0<\varepsilon<1$ and $\kappa>0$ such that any eigenvalue $\lambda \in \Lambda_{1, \delta} \equiv\left\{\lambda \in \Lambda_{1}|| \varepsilon^{2} \kappa^{2}+\lambda \mid \geq \delta\right\}$ of $(5.4)_{m},(5.6)_{N}$ satisfies $\left|\varepsilon^{2} \kappa^{2}+\lambda\right|<M_{c}$.

Proof. Setting $\psi=z_{m}$ in (7.20), we have

$$
\begin{aligned}
\left(\gamma+\lambda+\kappa^{2}\right)\left\|z_{m}\right\|_{L^{2}}^{2}+\left\|z_{m x}\right\|_{L^{2}}^{2}= & \frac{\left\langle S^{\varepsilon}(\lambda) z_{m}, \phi_{0}^{\varepsilon *}\right\rangle_{\mathbf{R}_{+}}}{\zeta_{0}^{\varepsilon}-\varepsilon^{2} \kappa^{2}-\lambda}\left\langle\phi_{0}^{\varepsilon}, z_{m}\right\rangle_{\mathbf{R}_{+}} \\
& +\left\langle\left(T^{\varepsilon}-\varepsilon^{2} \kappa^{2}-\lambda\right)^{\dagger} S^{\varepsilon}(\lambda) z_{m}, z_{m}\right\rangle_{\mathbf{R}_{+}} .
\end{aligned}
$$

For the real part of (7.21), it follows from (7.18) that

$$
\begin{aligned}
&(\gamma+\left.\operatorname{Re} \lambda+\kappa^{2}\right)\left\|z_{m}\right\|_{L^{2}}^{2}+\left\|z_{m x}\right\|_{L^{2}}^{2} \\
&= \operatorname{Re}\left\{\frac{\left\langle S^{\varepsilon}(\lambda) z_{m}, \phi_{0}^{\varepsilon *}\right\rangle_{\mathbf{R}_{+}}}{\zeta_{0}^{\varepsilon}-\varepsilon^{2} \kappa^{2}-\lambda}\left\langle\phi_{0}^{\varepsilon} z_{m}\right\rangle_{\mathbf{R}_{+}}+\left\langle\left(T^{\varepsilon}-\varepsilon^{2} \kappa^{2}-\lambda\right)^{\dagger} S^{\varepsilon}(\lambda) z_{m}, z_{m}\right\rangle_{\mathbf{R}_{+}}\right\} \\
& \leq \frac{\left|\left\langle S^{\varepsilon}(\lambda) z_{m}, \phi_{0}^{\varepsilon *}\right\rangle_{\mathbf{R}_{+}}\right|}{\left|\zeta_{0}^{\varepsilon}-\varepsilon^{2} \kappa^{2}-\lambda\right|}\left|\left\langle\phi_{0}^{\varepsilon}, z_{m}\right\rangle_{\mathbf{R}_{+}}\right|+\left|\left\langle\left(T^{\varepsilon}-\varepsilon^{2} \kappa^{2}-\lambda\right)^{\dagger} S^{\varepsilon}(\lambda) z_{m}, z_{m}\right\rangle_{\mathbf{R}_{+}}\right| \\
& \leq C\left\|z_{m}\right\|_{L^{2}}\left\{\left\|z_{m x}\right\|_{L^{2}}+\varepsilon\left(\gamma+\kappa^{2}+|\lambda|\right)\left\|z_{m}\right\|_{L^{2}}+\left\|z_{m}\right\|_{L^{2}}\right\} \\
& \quad \times\left\{\frac{1}{\left|\zeta_{0}^{\varepsilon}-\varepsilon^{2} \kappa^{2}-\lambda\right|}+\frac{1}{\left|\zeta_{1}^{\varepsilon}-\varepsilon^{2} \kappa^{2}-\lambda\right|}\right\}
\end{aligned}
$$

where $C$ is a positive constant independent of $0<\varepsilon<1$ and $\kappa>0$. Since $\left\|z_{m}\right\|_{L^{2}}=1$, the left-hand side of (7.22) is larger than the right side as $\left|\varepsilon^{2} \kappa^{2}+\operatorname{Re} \lambda\right| \rightarrow \infty$. This is a contradiction and therefore $\left|\varepsilon^{2} \kappa^{2}+\operatorname{Re} \lambda\right|<+\infty$.

On the other hand, we have

$$
\begin{aligned}
\operatorname{Im} \lambda\left\|z_{m}\right\|_{L^{2}}^{2}=\operatorname{Im} & \left\{\frac{\left\langle S^{\varepsilon}(\lambda) z_{m}, \phi_{0}^{\varepsilon *}\right\rangle_{\mathbf{R}_{+}}}{\zeta_{0}^{\varepsilon}-\varepsilon^{2} \kappa^{2}-\lambda}\left\langle\phi_{0}^{\varepsilon}, z_{m}\right\rangle_{\mathbf{R}_{+}}\right. \\
+ & \left.\left\langle\left(T^{\varepsilon}-\varepsilon^{2} \kappa^{2}-\lambda\right)^{\dagger} S^{\varepsilon}(\lambda) z_{m}, z_{m}\right\rangle_{\mathbf{R}_{+}}\right\}
\end{aligned}
$$

from (7.20). By using the same argument as above, we find that the left-hand side of the above equation has upper bound equal to the right-hand side in (7.22). Therefore, 
$\left\|z_{m x}\right\|_{L^{2}} \rightarrow \infty$ as $\operatorname{Im} \lambda \rightarrow \infty$. We note that there is a small positive constant $C_{1}$ independent of $0<\varepsilon<1$ and $\kappa>0$ such that

$$
\begin{aligned}
\left(\gamma+\kappa^{2}+\right. & \operatorname{Re} \lambda)+\left[1-C_{1}\left\{\frac{1}{\left|\zeta_{0}^{\varepsilon}-\varepsilon^{2} \kappa^{2}-\lambda\right|}+\frac{1}{\left|\zeta_{1}^{\varepsilon}-\varepsilon^{2} \kappa^{2}-\lambda\right|}\right\}\right]\left\|z_{m x}\right\|_{L^{2}}^{2} \\
& <\left(\frac{1}{C_{1}}+\varepsilon\left(\gamma+\kappa^{2}+|\lambda|\right)+1\right)\left\{\frac{1}{\left|\zeta_{0}^{\varepsilon}-\varepsilon^{2} \kappa^{2}-\lambda\right|}+\frac{1}{\left|\zeta_{1}^{\varepsilon}-\varepsilon^{2} \kappa^{2}-\lambda\right|}\right\} .
\end{aligned}
$$

As $|\operatorname{Im} \lambda| \rightarrow \infty$, the left-hand side term becomes larger than the right side for small $\varepsilon>0$, which is a contradiction. Hence, $|\operatorname{Im} \lambda|<\infty$, that is, there is a positive constant $M_{c}$ such that $\left|\varepsilon^{2} \kappa^{2}+\lambda\right|<M_{c}$ for each eigenvalue $\lambda \in \Lambda_{1, \delta}$.

Corollary 4. If $z_{m}$ is the eigenfunction corresponding to an eigenvalue $\lambda \in \Lambda_{1, \delta}$ satisfying $\left|\varepsilon^{2} \kappa^{2}+\lambda\right|<M_{c}$ of $(5.4)_{m},(5.6)_{N}$, then there is a positive constant $\hat{C}$ such that

$$
1 \leq\left\|z_{m}\right\|_{H^{2}} \leq \hat{C} \text {. }
$$

Proof. From (7.21), there is a positive constant $C_{3}$ satisfying

$$
\begin{aligned}
\left\|z_{m x}\right\|_{L^{2}}^{2} \leq( & \left.+\kappa^{2}+|\lambda|\right)+\left|\frac{\left\langle S^{\varepsilon}(\lambda) z_{m}, \phi_{0}^{\varepsilon *}\right\rangle_{\mathbf{R}_{+}}}{\zeta_{0}^{\varepsilon}-\varepsilon^{2} \kappa^{2}-\lambda}\left\langle\phi_{0}^{\varepsilon}, z_{m}\right\rangle_{\mathbf{R}_{+}}\right| \\
& +\left|\left\langle\left(T^{\varepsilon}-\varepsilon^{2} \kappa^{2}-\lambda\right)^{\dagger} S^{\varepsilon}(\lambda) z_{m}, z_{m}\right\rangle_{\mathbf{R}_{+}}\right| \\
\leq( & \left.+\kappa^{2}+|\lambda|\right)+C_{3}\left\{\left\|z_{m x}\right\|_{L^{2}}+\varepsilon\left(\gamma+\kappa^{2}+|\lambda|\right)+1\right\} \\
& \times\left\{\frac{1}{\left|\zeta_{0}^{\varepsilon}-\varepsilon^{2} \kappa^{2}-\lambda\right|}+\frac{1}{\left|\zeta_{1}^{\varepsilon}-\varepsilon^{2} \kappa^{2}-\lambda\right|}\right\} .
\end{aligned}
$$

By using $\left\|z_{m}\right\|_{L^{2}}=1$, we have

$$
\begin{aligned}
& {\left[1-\frac{C_{3} C_{4}}{2}\left\{\frac{1}{\left|\zeta_{0}^{\varepsilon}-\varepsilon^{2} \kappa^{2}-\lambda\right|}+\frac{1}{\left|\zeta_{1}^{\varepsilon}-\varepsilon^{2} \kappa^{2}-\lambda\right|}\right\}\right]\left\|z_{m x}\right\|_{L^{2}}^{2} \leq \gamma+\kappa^{2}+|\lambda|} \\
& \quad+C_{3}\left\{\frac{1}{2 C_{4}}+\varepsilon\left(\gamma+\kappa^{2}+|\lambda|\right)+1\right\}\left\{\frac{1}{\left|\zeta_{0}^{\varepsilon}-\varepsilon^{2} \kappa^{2}-\lambda\right|}+\frac{1}{\left|\zeta_{1}^{\varepsilon}-\varepsilon^{2} \kappa^{2}-\lambda\right|}\right\}
\end{aligned}
$$

for a small positive constant $C_{4}$. Thus, it turns out that there is a positive constant $C_{5}$ satisfying $\left\|z_{m x}\right\|_{L^{2}} \leq C_{5}$. Moreover, it follows from (7.19) that $\left\|z_{m x x}\right\|_{L^{2}} \leq C_{6}\left\|z_{m}\right\|_{H^{1}}$ with some positive constant $C_{6}$. Therefore, there is a positive constant $\hat{C}$ satisfying $1 \leq\left\|z_{m}\right\|_{H^{2}} \leq \hat{C}$.

To show the distribution of the eigenvalues of $(5.4)_{m},(5.6)_{N}$ in $\left\{\lambda \in \Lambda_{1, \delta} \mid\right.$ $\left.\left|\varepsilon^{2} \kappa^{2}+\lambda\right|<M_{c}\right\}$, we need the two lemmas:

Lemma 8. [19, Lemma 2.2] Let $F(u, v)$ be a smooth function of $u$ and $v$. Then, for $F^{\varepsilon}=F\left(u^{\varepsilon}(x), v^{\varepsilon}(x)\right)$ and $F^{*}=F\left(U^{*}(x), V^{*}(x)\right)$

$$
\left(T^{\varepsilon}-\lambda\right)^{\dagger}\left(F^{\varepsilon} h\right) \rightarrow \frac{F^{*} h}{f^{\prime}\left(W_{0}\right)-\lambda} \quad \text { strongly in the } L^{2} \text {-sense as } \varepsilon \downarrow 0
$$

for any $h \in L_{\rho}^{2}\left(\mathbf{R}_{+}\right) \cap L^{\infty}\left(\mathbf{R}_{+}\right)$. Moreover, for any finite $\lambda \in \Lambda_{1}$, $\left(T^{\varepsilon}-\lambda\right)^{\dagger}\left(\varepsilon u_{x}^{\varepsilon} \cdot\right) \rightarrow 0$ uniformly on any bounded set in $H^{1}\left(\mathbf{R}_{+}\right) \cap L^{\infty}\left(\mathbf{R}_{+}\right)$as $\varepsilon \downarrow 0$.

Lemma 9. There is an $\varepsilon_{0}>0$ such that any $\lambda \in \Lambda_{1, \delta}$ satisfying $\left|\varepsilon^{2} \kappa^{2}+\lambda\right|<M_{c}$ is not an eigenvalue of $(5.4)_{m},(5.6)_{N}$ for any $0<\varepsilon<\varepsilon_{0}$. 
Proof. Let $\lambda$ be an eigenvalue of $(5.4)_{m},(5.6)_{N}$. From Corollaries 3 and 4 , we find positive constants $C_{1}, C_{2}, C_{3}$, and $C_{4}$ independent of $\varepsilon$ and $\kappa$ such that

$$
\left|\left\langle\phi_{0}^{\varepsilon}, z_{m}\right\rangle_{\mathbf{R}_{+}}\right| \leq\left\|z_{m}\right\|_{L^{\infty}} C_{1} \sqrt{\varepsilon} \leq C_{2} \sqrt{\varepsilon}
$$

and

$$
\left|\left\langle S^{\varepsilon}(\lambda) z_{m}, \phi_{0}^{\varepsilon *}\right\rangle_{\mathbf{R}_{+}}\right| \leq\left\|S^{\varepsilon}(\lambda) z_{m}\right\|_{L^{2}}\left\|\phi_{0}^{\varepsilon *}\right\|_{L^{2}} \leq C_{3}\left\|S^{\varepsilon}(\lambda) z_{m}\right\|_{L^{2}}<C_{4}(1+\varepsilon|\lambda|)
$$

for small $\varepsilon>0$. By using Corollary 4 and Lemma 8, we see that the left side of (7.21) is larger than the right side for small $\varepsilon>0$, which is a contradiction.

We thus find that there is no eigenvalue of $(5.4)_{m},(5.6)_{N}$ in $\left\{\lambda \in \Lambda_{1}|| \varepsilon^{2} \kappa^{2}+\lambda \mid>\right.$ $\delta\}$. Since the same argument as the above also can be used for the eigenvalue of $(5.4)_{m},(5.6)_{D}$, Proposition 1 is completely proved.

Proof of Theorem 2. Hereafter, we may assume that there exists a positive function $\delta(\varepsilon)$ with $\lim _{\varepsilon \downarrow 0} \delta(\varepsilon)=0$ such that

$$
\left|\varepsilon^{2} \kappa^{2}+\lambda\right|<\delta(\varepsilon)
$$

We now consider the distribution of eigenvalues satisfying (7.23). To do so, we define the bilinear form

$$
\hat{B}^{\kappa, \lambda}\left(z^{1}, z^{2}\right)=\left\langle z_{x}^{1}, z_{x}^{2}\right\rangle_{\mathbf{R}_{+}}+\left(\gamma+\kappa^{2}+\lambda\right)\left\langle z^{1}, z^{2}\right\rangle_{\mathbf{R}_{+}}
$$

for $z^{1}, z^{2} \in H_{N}^{1}\left(\mathbf{R}_{+}\right)$. It is easy to see that there are two positive constants $C_{5}$ and $C_{6}$ such that

$$
\left|\hat{B}^{\kappa, \lambda}\left(z^{1}, z^{2}\right)\right| \leq C_{5}\left\|z^{1}\right\|_{H^{1}}\left\|z^{2}\right\|_{H^{1}}
$$

and

$$
\left|\hat{B}^{\kappa, \lambda}(z, z)\right| \geq C_{6}\|z\|_{H^{1}}^{2}
$$

for any $\lambda$ and $\kappa$ satisfying (7.23).

By the Lax-Milgram theorem, we find that for any $h \in H^{-1}\left(\mathbf{R}_{+}\right)$, there exists $z \in H_{N}^{1}\left(\mathbf{R}_{+}\right)$such that

$$
\hat{B}^{\kappa, \lambda}(z, \psi)=\langle h, \psi\rangle_{\mathbf{R}_{+}} \quad \text { for any } \psi \in H_{N}^{1}\left(\mathbf{R}_{+}\right) .
$$

Then a linear operator $K^{\kappa, \lambda}$ from $H^{-1}\left(\mathbf{R}_{+}\right)$to $H_{N}^{1}\left(\mathbf{R}_{+}\right)$can be defined by $z=K^{\kappa, \lambda} h$.

Lemma 10. For any $\lambda$ and $\kappa$ satisfying (7.23), $K^{\kappa, \lambda}$ satisfies an estimate $\left\|K^{\kappa, \lambda}\right\| \leq$ $\frac{1}{C_{6}}$ where $\|\cdot\|$ means the operator norm from $H^{-1}\left(\mathbf{R}_{+}\right)$to $H_{N}^{1}\left(\mathbf{R}_{+}\right)$.

By (7.20) and (7.24), it holds that for any $\psi \in H_{N}^{1}\left(\mathbf{R}_{+}\right)$,

$$
\hat{B}^{\kappa, \lambda}\left(z_{m}, \psi\right)=\frac{\left\langle S^{\varepsilon}(\lambda) z_{m}, \phi_{0}^{\varepsilon *}\right\rangle_{\mathbf{R}_{+}}}{\zeta_{0}^{\varepsilon}-\varepsilon^{2} \kappa^{2}-\lambda}\left\langle\phi_{0}^{\varepsilon}, \psi\right\rangle_{\mathbf{R}_{+}}+\left\langle\left(T^{\varepsilon}-\varepsilon^{2} \kappa^{2}-\lambda\right)^{\dagger} S^{\varepsilon}(\lambda) z_{m}, \psi\right\rangle_{\mathbf{R}_{+}}
$$

where $z_{m}$ is the eigenfunction corresponding to the eigenvalue $\lambda$. Therefore, it follows from Lemma 10 that

$$
\begin{aligned}
z_{m} & =\frac{\left\langle S^{\varepsilon}(\lambda) z_{m}, \phi_{0}^{\varepsilon *}\right\rangle_{\mathbf{R}_{+}}}{\zeta_{0}^{\varepsilon}-\varepsilon^{2} \kappa^{2}-\lambda} K^{\kappa, \lambda}\left(\phi_{0}^{\varepsilon}\right)+K^{\kappa, \lambda}\left(\left(T^{\varepsilon}-\varepsilon^{2} \kappa^{2}-\lambda\right)^{\dagger} S^{\varepsilon}(\lambda) z_{m}\right) \\
& =\frac{\left\langle S^{\varepsilon}(\lambda) z_{m}, \phi_{0}^{\varepsilon *} / \sqrt{\varepsilon}\right\rangle_{\mathbf{R}_{+}}}{\left(\zeta_{0}^{\varepsilon}-\varepsilon^{2} \kappa^{2}-\lambda\right) / \varepsilon} K^{\kappa, \lambda}\left(\frac{\phi_{0}^{\varepsilon}}{\sqrt{\varepsilon}}\right)+K^{\kappa, \lambda}\left(\left(T^{\varepsilon}-\varepsilon^{2} \kappa^{2} \lambda\right)^{\dagger} S^{\varepsilon}(\lambda) z_{m}\right)
\end{aligned}
$$

Put

$$
z_{m}=\alpha K^{\kappa, \lambda}\left(\frac{\phi_{0}^{\varepsilon}}{\sqrt{\varepsilon}}\right)+K^{\kappa, \lambda}(b)
$$


with $\left\langle b, \phi_{0}^{\varepsilon *}\right\rangle_{\mathbf{R}_{+}}=0$. Then, (7.25) and (7.26) imply that

$$
\begin{aligned}
& \frac{\alpha\left(\zeta_{0}^{\varepsilon}-\varepsilon^{2} \kappa^{2}-\lambda\right)}{\varepsilon}=\alpha\left\langle S^{\varepsilon}(\lambda) K^{\kappa, \lambda}\left(\frac{\phi_{0}^{\varepsilon}}{\sqrt{\varepsilon}}\right), \frac{\phi_{0}^{\varepsilon *}}{\sqrt{\varepsilon}}\right\rangle_{\mathbf{R}_{+}},+\left\langle S^{\varepsilon}(\lambda) K^{\kappa, \lambda}(b), \frac{\phi_{0}^{\varepsilon *}}{\sqrt{\varepsilon}}\right\rangle_{\mathbf{R}_{+}}, \\
& b=\left(T^{\varepsilon}-\varepsilon^{2} \kappa^{2}-\lambda\right)^{\dagger} S^{\varepsilon}(\lambda)\left(\alpha K^{\kappa, \lambda}\left(\frac{\phi_{0}^{\varepsilon}}{\sqrt{\varepsilon}}\right)+K^{\kappa, \lambda}(b)\right) .
\end{aligned}
$$

Then, the second equation of (7.27) is rewritten as

$$
\left[I-\left(T^{\varepsilon}-\varepsilon^{2} \kappa^{2}-\lambda\right)^{\dagger} S^{\varepsilon}(\lambda) K^{\kappa \lambda}\right] b=\alpha\left(T^{\varepsilon}-\varepsilon^{2} \kappa^{2}-\lambda\right)^{\dagger} S^{\varepsilon}(\lambda) K^{\kappa, \lambda}\left(\frac{\phi_{0}^{\varepsilon}}{\sqrt{\varepsilon}}\right) .
$$

We remark that $K^{\kappa, \lambda}$ is also a bounded operator from $L^{2}\left(\mathbf{R}_{+}\right)$to $H_{N}^{2}\left(\mathbf{R}_{+}\right)$. Therefore, $K^{\kappa, \lambda}\left(\frac{\phi_{0}^{\varepsilon}}{\sqrt{\varepsilon}}\right) \in H_{N}^{2}\left(\mathbf{R}_{+}\right)$because $\frac{\phi^{\varepsilon_{0}}}{\sqrt{\varepsilon}} \in L^{2}\left(\mathbf{R}_{+}\right)$holds for small $\varepsilon>0$. Moreover,

$$
\left\|S^{\varepsilon}(\lambda) K^{\kappa, \lambda}\left(\frac{\phi_{0}^{\varepsilon}}{\sqrt{\varepsilon}}\right)\right\|_{L_{\rho}^{2}}=O(\varepsilon)
$$

and

$$
\left\|\left(T^{\varepsilon}-\varepsilon^{2} \kappa^{2}-\lambda\right)^{\dagger} S^{\varepsilon}(\lambda) K^{\kappa, \lambda}\right\|_{L^{2} \rightarrow L_{\rho}^{2}}=O(\varepsilon) \quad \text { for small } \varepsilon>0
$$

Therefore, the operator $I-\left(T^{\varepsilon}-\varepsilon^{2} \kappa^{2}-\lambda\right)^{\dagger} S^{\varepsilon}(\lambda) K^{\kappa, \lambda}$ has a bounded inverse from $L^{2}\left(\mathbf{R}_{+}\right)$to $L^{2}\left(\mathbf{R}_{+}\right)$. We have $b=\left[I-\left(T^{\varepsilon}-\varepsilon^{2} \kappa^{2}-\lambda\right)^{\dagger} S^{\varepsilon}(\lambda) K^{\kappa, \lambda}\right]^{-1} \alpha S^{\varepsilon}(\lambda) K^{\kappa, \lambda}\left(\frac{\phi_{0}^{\varepsilon}}{\sqrt{\varepsilon}}\right)=$ $O(\varepsilon)$ in $L^{2}\left(\mathbf{R}_{+}\right)$, which implies that $K^{\kappa, \lambda}(b)=O(\varepsilon)$ in $H_{N}^{2}\left(\mathbf{R}_{+}\right)$for small $\varepsilon>0$. If $\alpha=0$, we know $b=0$, that is, $\left(w_{m}, z_{m}\right)=(0,0)$. This contradicts the fact that $\left(w_{m}, z_{m}\right)$ is an eigenfunction, so that $\alpha \neq 0$ holds.

Next, we consider the limiting form of the first equation in (7.27) when $\varepsilon$ tends to 0 .

Lemma 11. [19, Lemma 2.3]

$$
\begin{gathered}
\lim _{\varepsilon \downarrow 0} \frac{\phi_{0}^{\varepsilon}}{\sqrt{\varepsilon}}=\kappa^{*} \delta_{0} \quad \text { and } \quad \lim _{\varepsilon \downarrow 0} \frac{\phi_{0}^{\varepsilon *}}{\sqrt{\varepsilon}}=\kappa^{*} \gamma^{*} \delta_{0} \quad \text { in the } H^{-1}\left(\mathbf{R}_{+}\right) \text {-sense } \\
\lim _{\varepsilon \downarrow 0} \sqrt{\varepsilon} u_{x}^{\varepsilon} \chi^{\prime}\left(v^{\varepsilon}\right) \phi_{0}^{\varepsilon *}=-\kappa^{*} \gamma^{*} \chi^{\prime}\left(\sigma^{*}\right)\left\langle\left(W_{0 \xi}\right)^{2}, e^{c \xi}\right\rangle_{\mathbf{R}} \delta_{0} \quad \text { in the } H^{-1}\left(\mathbf{R}_{+}\right) \text {-sense, }
\end{gathered}
$$

and

$\lim _{\varepsilon \downarrow 0} \sqrt{\varepsilon} u_{x}^{\varepsilon} \chi^{\prime \prime}\left(v^{\varepsilon}\right) v_{x}^{\varepsilon} \phi_{0}^{\varepsilon *}=-\kappa^{*} \gamma^{*} \chi^{\prime \prime}\left(\sigma^{*}\right) v_{x}^{*}\left\langle\left(W_{0 \xi}\right)^{2}, e^{c \xi}\right\rangle_{\mathbf{R}} \delta_{0} \quad$ in the $H^{-1}\left(\mathbf{R}_{+}\right)$-sense where $\delta_{0}$ is the Dirac $\delta$-function at $x=\eta^{*}$.

By Corollary 3, we have a positive constant $C$ such that

$$
\left|\left\langle S^{\varepsilon}(\lambda) K^{\kappa, \lambda}(b), \phi_{0}^{\varepsilon *}\right\rangle_{\mathbf{R}_{+}}\right| \leq C \sqrt{\varepsilon}\left\|\phi_{0}^{\varepsilon *}\right\|_{L^{1}\left(\mathbf{R}_{+}\right)}=C L^{*}(\varepsilon) \varepsilon
$$

for small $\varepsilon>0$ where $C$ is a constant independent on $\kappa$.

We first assume that $\kappa$ is large to be $O\left(\varepsilon^{-\frac{1}{2}}\right)$ for small $\varepsilon>0$. Then, the following equation is obtained by $(7.27),(7.28)$ :

$$
\alpha\left(\hat{\zeta}_{0}^{*}-\varepsilon \kappa^{2}-\tau_{N}^{*}\right)=\alpha \lim _{\varepsilon \downarrow 0}\left\langle S^{\varepsilon}(\varepsilon \tau) K^{\kappa, \varepsilon \tau}\left(\frac{\phi_{0}^{\varepsilon}}{\sqrt{\varepsilon}}\right), \frac{\phi_{0}^{\varepsilon *}}{\sqrt{\varepsilon}}\right\rangle_{\mathbf{R}_{+}}
$$

for $\lambda=\varepsilon \tau$ where $\lim _{\varepsilon \downarrow 0} \frac{\zeta_{0}^{\varepsilon}}{\varepsilon}=\hat{\zeta}_{0}^{*}$ and $\lim _{\varepsilon \downarrow 0} \tau=\tau_{N}^{*}$. Putting $\hat{z}=\alpha K^{\kappa, \varepsilon \tau}\left(\frac{\phi_{0}^{\varepsilon}}{\sqrt{\varepsilon}}\right)$, we have

$$
\left\langle\hat{z}_{x}, \psi_{x}\right\rangle_{\mathbf{R}_{+}}+\left(\gamma+\kappa^{2}+\varepsilon \tau\right)\langle\hat{z}, \psi\rangle_{\mathbf{R}_{+}}=\alpha\left\langle\frac{\phi_{0}^{\varepsilon}}{\sqrt{\varepsilon}}, \psi\right\rangle_{\mathbf{R}_{+}} \quad \text { for any } \quad \psi \in H_{N}^{1}\left(\mathbf{R}_{+}\right)
$$


by the definition of $K^{\kappa, \varepsilon \tau}$. Thus, it follows from the above equation and Lemma 11 that $\hat{z}^{*}=\lim _{\varepsilon \downarrow 0} \hat{z}$ satisfies $\hat{z}^{*} \in C^{2}\left(\left(0, \eta^{*}\right) \cup\left(\eta^{*}, \infty\right)\right)$,

$$
\hat{z}_{x}^{*}\left(\eta^{*}-0\right)-\hat{z}_{x}^{*}\left(\eta^{*}+0\right)=\alpha \kappa^{*}
$$

and

$$
\begin{aligned}
& \hat{z}_{x x}^{*}-\left(\gamma+\kappa^{2}\right) \hat{z}^{*}=0 \quad \text { in } \quad\left(0, \eta^{*}\right) \cup\left(\eta^{*}, \infty\right), \\
& \hat{z}^{*}\left(\eta^{*}\right)=\nu, \quad \hat{z}_{x}^{*}(0)=0, \quad \hat{z}^{*}(\infty)=0,
\end{aligned}
$$

for some positive constant $\nu$ satisfying $\left\|\hat{z}^{*}\right\|_{L^{2}}=1$. Then, the solution $\hat{z}^{*}$ of the above problem is represented by

$$
\hat{z}^{*}(x)= \begin{cases}\nu \frac{e^{\sqrt{\gamma+\kappa^{2}} x}+e^{-\sqrt{\gamma+\kappa^{2}} x}}{e^{\sqrt{\gamma+\kappa^{2}} \eta^{*}}+e^{-\sqrt{\gamma+\kappa^{2}} \eta^{*}}}, & x \in\left(0, \eta^{*}\right), \\ \nu e^{-\sqrt{\gamma+\kappa^{2}}\left(x-\eta^{*}\right)}, & x \in\left(\eta^{*}, \infty\right) .\end{cases}
$$

Thus, we have

$$
\begin{aligned}
{\left[\hat{z}_{x}^{*}\right] } & \equiv \hat{z}_{x}^{*}\left(\eta^{*}+0\right)-\hat{z}_{x}^{*}\left(\eta^{*}-0\right) \\
& =\nu \sqrt{\gamma+\kappa^{2}}\left\{-1-\frac{e^{\sqrt{\gamma+\kappa^{2}} \eta^{*}}-e^{-\sqrt{\gamma+\kappa^{2}} \eta^{*}}}{e^{\sqrt{\gamma+\kappa^{2}} \eta^{*}}+e^{-\sqrt{\gamma+\kappa^{2}} \eta^{*}}}\right\} \\
& =-\frac{2 \nu \sqrt{\gamma+\kappa^{2}}}{1+e^{-2 \sqrt{\gamma+\kappa^{2}} \eta^{*}}} .
\end{aligned}
$$

Since $K^{\kappa, \varepsilon \tau}(b)=O(\varepsilon)$ in $H_{N}^{2}\left(\mathbf{R}_{+}\right)$for small $\varepsilon>0$, it follows from (7.26) that $\hat{z}_{m}^{*}=$ $\lim _{\varepsilon \downarrow 0} z_{m}=\hat{z}^{*}$ in $H^{1}\left(\mathbf{R}_{+}\right)$. By using the first equation in (7.27) and Lemma 11, we have

$$
\begin{aligned}
\alpha\left(\hat{\zeta}_{0}^{*}-\varepsilon \kappa^{2}-\tau\right)=- & k \gamma^{*} \kappa^{*}\left[\chi^{\prime \prime}\left(\sigma^{*}\right) v_{x}^{*} z_{m}^{*}\left(\eta^{*}\right)\left\langle\left(W_{0 \xi}\right)^{2}, e^{c \xi}\right\rangle_{\mathbf{R}}\right. \\
& +\chi^{\prime}\left(\sigma^{*}\right)\left\{z_{m x}^{*}\left(\eta^{*}-0\right)\left\langle\left(W_{0 \xi}\right)^{2}, e^{c \xi}\right\rangle_{\mathbf{R}_{-}}\right. \\
& \left.\left.+z_{m x}^{*}\left(\eta^{*}+0\right)\left\langle\left(W_{0 \xi}\right)^{2}, e^{c \xi}\right\rangle_{\mathbf{R}_{+}}\right\}\right]
\end{aligned}
$$

We next consider the case of the Dirichlet boundary conditions. The same argument as the one for the Neumann boundary conditions implies that the eigenfunction $\hat{z}_{D}^{*}$ corresponding to the principal eigenvalue with the Dirichlet boundary conditions satisfies

$$
\begin{array}{ll}
\hat{z}_{D x x}^{*}-\left(\gamma+\kappa^{2}\right) \hat{z}_{D}^{*}=0 & \text { in }\left(0, \eta^{*}\right) \cup\left(\eta^{*}, \infty\right) \\
\hat{z}_{D}^{*}\left(\eta^{*}\right)=\nu, \quad \hat{z}_{D}^{*}(0)=0, & \hat{z}_{D}^{*}(\infty)=0
\end{array}
$$

and

$$
\hat{z}_{D x}^{*}\left(\eta^{*}-0\right)-\hat{z}_{D x}^{*}\left(\eta^{*}+0\right)=\alpha_{D} \kappa^{*}
$$

where $\alpha_{D}$ satisfies (7.26) corresponding to the Dirichlet boundary condition. The solution $\hat{z}_{D}^{*}(x)$ of (7.32) is represented by

$$
\hat{z}_{D}^{*}(x)= \begin{cases}\nu \frac{e^{\sqrt{\gamma+\kappa^{2}} x}-e^{-\sqrt{\gamma+\kappa^{2}} x}}{e^{\sqrt{\gamma+\kappa^{2}} \eta^{*}}-e^{-\sqrt{\gamma+\kappa^{2}} \eta^{*}}}, & x \in\left(0, \eta^{*}\right), \\ \nu e^{-\sqrt{\gamma+\kappa^{2}}\left(x-\eta^{*}\right)}, & x \in\left(\eta^{*}, \infty\right) .\end{cases}
$$


STABILITY OF PLANAR EQUILIBRIUM SOLUTIONS IN A CHEMOTAXIS MODEL 427

Thus, we obtain

$$
\begin{aligned}
{\left[z_{D x}^{*}\right] } & =z_{D x}^{*}\left(\eta^{*}+0\right)-z_{D x}^{*}\left(\eta^{*}-0\right) \\
& =\nu \sqrt{\gamma+\kappa^{2}}\left\{-1-\frac{e^{\sqrt{\gamma+\kappa^{2}} \eta^{*}}+e^{-\sqrt{\gamma+\kappa^{2}} \eta^{*}}}{e^{\sqrt{\gamma+\kappa^{2}} \eta^{*}}-e^{-\sqrt{\gamma+\kappa^{2}} \eta^{*}}}\right\} \\
& =-\frac{2 \nu \sqrt{\gamma+\kappa^{2}}}{1-e^{-2 \sqrt{\gamma+\kappa^{2}} \eta^{*}}} .
\end{aligned}
$$

Summarizing the above results, we have

$$
\frac{\nu}{\alpha \kappa^{*}}=\frac{1+e^{-2 \sqrt{\gamma+\kappa^{2}} \eta^{*}}}{2 \sqrt{\gamma+\kappa^{2}}} \text { and } \frac{\nu}{\alpha_{D} \kappa^{*}}=\frac{1-e^{-2 \sqrt{\gamma+\kappa^{2}} \eta^{*}}}{2 \sqrt{\gamma+\kappa^{2}}}
$$

It follows from (7.31) that

$$
\begin{aligned}
\tau=\hat{\zeta}_{0}^{*}-\varepsilon & \kappa^{2}+\frac{k \nu \gamma^{*} \kappa^{*}}{\alpha}\left[-\frac{\sigma^{*} \chi^{\prime \prime}\left(\sigma^{*}\right) \sqrt{\gamma}}{\gamma^{*}\left(\kappa^{*}\right)^{2}}+\chi^{\prime}\left(\sigma^{*}\right) \sqrt{\gamma+\kappa^{2}}\right. \\
& \left.\times\left\{\left(\frac{1-e^{-2 \sqrt{\gamma+\kappa^{2}} \eta^{*}}}{1+e^{-2 \sqrt{\gamma+\kappa^{2}} \eta^{*}}}+1\right)\left\langle\left(W_{0 \xi}\right)^{2}, e^{c \xi}\right\rangle_{\mathbf{R}_{-}}-\frac{1}{\gamma^{*}\left(\kappa^{*}\right)^{2}}\right\}\right] \\
= & \hat{\zeta}_{0}^{*}-\varepsilon \kappa^{2}+\frac{k \nu}{\alpha \kappa^{*}}\left[-\sigma^{*} \chi^{\prime \prime}\left(\sigma^{*}\right) \sqrt{\gamma}+\chi^{\prime}\left(\sigma^{*}\right) \sqrt{\gamma+\kappa^{2}}\right. \\
& \left.\times\left\{\frac{2 \gamma^{*}\left(\kappa^{*}\right)^{2}}{1+e^{-2 \sqrt{\gamma+\kappa^{2}} \eta^{*}}}\left\langle\left(W_{0 \xi}\right)^{2}, e^{c \xi}\right\rangle_{\mathbf{R}_{-}}-1\right\}\right] \\
=\hat{\zeta}_{0}^{*}-\varepsilon & +\frac{k\left(1+\kappa^{2}-2 \sqrt{\gamma+\kappa^{2}} \eta^{*}\right)}{2 \sqrt{\gamma+\kappa^{2}}}\left[-\sigma^{*} \chi^{\prime \prime}\left(\sigma^{*}\right) \sqrt{\gamma}\right. \\
& \left.+\chi^{\prime}\left(\sigma^{*}\right) \sqrt{\gamma+\kappa^{2}}\left\{\frac{2 \gamma^{*}\left(\kappa^{*}\right)^{2}}{1+e^{-2 \sqrt{\gamma+\kappa^{2}} \eta^{*}}}\left\langle\left(W_{0 \xi}\right)^{2}, e^{c \xi}\right\rangle_{\mathbf{R}_{-}}-1\right\}\right] \\
= & \hat{\zeta}_{0}^{*}-\varepsilon \kappa^{2}-k\left[\frac{\left(1+e^{-2 \sqrt{\gamma+\kappa^{2}} \eta^{*}}\right) \sigma^{*} \chi^{\prime \prime}\left(\sigma^{*}\right) \sqrt{\gamma}}{2 \sqrt{\gamma+\kappa^{2}}}-\chi^{\prime}\left(\sigma^{*}\right)\left\langle\left(W_{0 \xi}\right)^{2}, e^{c \xi}\right\rangle_{\mathbf{R}_{-}} \gamma^{*}\left(\kappa^{*}\right)^{2}\right. \\
& \left.+\chi^{\prime}\left(\sigma^{*}\right) \frac{1+e^{-2 \sqrt{\gamma+\kappa^{2}} \eta^{*}}}{2}\right] .
\end{aligned}
$$

From Lemma 5 and (4.3), it is easy to see that

$$
\begin{aligned}
& \tau_{N}= \\
&=-\varepsilon \kappa^{2}+k\left[\frac{\sigma^{*} \chi^{\prime \prime}\left(\sigma^{*}\right)}{2}\left\{1-e^{-2 \sqrt{\gamma} \eta^{*}}-\frac{\sqrt{\gamma}}{\sqrt{\gamma+\kappa^{2}}}\left(1+e^{-2 \sqrt{\gamma+\kappa^{2}} \eta^{*}}\right)\right\}\right. \\
&\left.\quad \quad-\frac{e^{-2 \sqrt{\gamma} \eta^{*}}+e^{-2 \sqrt{\gamma+\kappa^{2}} \eta^{*}}}{2} \chi^{\prime}\left(\sigma^{*}\right)\right] \\
&=A^{v}(\kappa) .
\end{aligned}
$$


Since (7.31) also is satisfied by $\hat{z}_{D}^{*}$ and $\alpha_{D}$, it similarly follows from (7.32), (7.33), and (4.4) that

$$
\begin{aligned}
& \tau_{D}= \tau \\
&= \hat{\zeta}_{0}^{*}-\varepsilon \kappa^{2}+\frac{k \nu \gamma^{*} \kappa^{*}}{\alpha_{D}}\left[-\frac{\sigma^{*} \chi^{\prime \prime}\left(\sigma^{*}\right) \sqrt{\gamma}}{\gamma^{*}\left(\kappa^{*}\right)^{2}}+\chi^{\prime}\left(\sigma^{*}\right) \sqrt{\gamma+\kappa^{2}}\right. \\
&\left.\times\left\{\frac{2}{1-e^{-2 \sqrt{\gamma+\kappa^{2}} \eta^{*}}}\left\langle\left(W_{0 \xi}\right)^{2}, e^{c \xi}\right\rangle_{\mathbf{R}_{-}}-\frac{1}{\gamma^{*}\left(\kappa^{*}\right)^{2}}\right\}\right] \\
&=-\varepsilon \kappa^{2}+k\left[\frac{\sigma^{*} \chi^{\prime \prime}\left(\sigma^{*}\right)}{2}\left\{1-e^{-2 \sqrt{\gamma} \eta^{*}}-\frac{\sqrt{\gamma}}{\sqrt{\gamma+\kappa^{2}}}\left(1-e^{-2 \sqrt{\gamma+\kappa^{2}} \eta^{*}}\right)\right\}\right. \\
&\left.\quad-\frac{e^{-2 \sqrt{\gamma} \eta^{*}}-e^{-2 \sqrt{\gamma+\kappa^{2}} \eta^{*}}}{2} \chi^{\prime}\left(\sigma^{*}\right)\right] \\
&=A^{z}(\kappa) .
\end{aligned}
$$

Define $\mathcal{F}(\varepsilon, \tau, \kappa)$ by

$$
\begin{aligned}
\mathcal{F}(\varepsilon, \tau, \kappa)=\tau & +\varepsilon \kappa^{2}-\hat{\zeta}_{0}(\varepsilon)+\left\langle S^{\varepsilon}(\varepsilon \tau) K^{\kappa, \varepsilon \tau}\left(\frac{\phi_{0}^{\varepsilon}}{\sqrt{\varepsilon}}\right), \frac{\phi_{0}^{\varepsilon *}}{\sqrt{\varepsilon}}\right\rangle_{\mathbf{R}_{+}} \\
& +\frac{1}{\alpha}\left\langle S^{\varepsilon}(\varepsilon \tau) K^{\kappa, \varepsilon \tau}(b) \frac{\phi_{0}^{\varepsilon}}{\sqrt{\varepsilon}}\right\rangle .
\end{aligned}
$$

We find that the critical eigenvalues of $(5.4)_{m},(5.6)_{N}$ satisfy $\mathcal{F}(\varepsilon, \tau, \kappa)=0$. (7.27) implies

$$
\mathcal{F}(\varepsilon, \tau, \kappa)=\tau+\varepsilon \kappa^{2}-\hat{\zeta}_{0}(\varepsilon)+\left\langle S^{\varepsilon}(\varepsilon \tau) K^{\kappa, \varepsilon \tau}\left(\frac{\phi_{0}^{\varepsilon}}{\sqrt{\varepsilon}}\right), \frac{\phi_{0}^{\varepsilon *}}{\sqrt{\varepsilon}}\right\rangle_{\mathbf{R}_{+}}+R(\varepsilon)
$$

where $R(\varepsilon)=O(\varepsilon)$ for small $\varepsilon>0$. Then, it follows from Lemmas 9 and 10 that

$$
\begin{aligned}
\mathcal{F}(0, \tau, \kappa)=\tau & +\mu^{*}-\zeta_{0}^{*}-k \gamma^{*}\left(\kappa^{*}\right)^{2}\left[\chi^{\prime \prime}\left(\sigma^{*}\right) v_{x}^{*}\left\langle\left(W_{0 \xi}\right)^{2}, e^{c \xi}\right\rangle_{\mathbf{R}}\left\langle K^{\kappa, 0}\left(\delta_{0}\right), \delta_{0}\right\rangle_{\mathbf{R}_{+}}\right. \\
& +\chi^{\prime}\left(\sigma^{*}\right)\left\{\left(K^{\kappa, 0}\left(\delta_{0}\right)\right)_{x}\left(\eta^{*}-0\right)\left\langle\left(W_{0 \xi}\right)^{2}, e^{c \xi}\right\rangle_{\mathbf{R}_{-}}\right. \\
& \left.\left.+\left(K^{\kappa, 0}\left(\delta_{0}\right)\right)_{x}\left(\eta^{*}+0\right)\left\langle\left(W_{0 \xi}\right)^{2}, e^{c \xi}\right\rangle_{\mathbf{R}_{+}}\right\}\right]
\end{aligned}
$$

where $\lim _{\varepsilon \downarrow 0} \varepsilon \kappa^{2}=\mu^{*}$. Therefore, it holds that $\mathcal{F}(0, \tau, \kappa)=0$ if and only if

$$
\begin{aligned}
\tau=\zeta_{0}^{*} & -\mu^{*}+k \gamma^{*}\left(\kappa^{*}\right)^{2}\left[\chi^{\prime \prime}\left(\sigma^{*}\right) v_{x}^{*}\left\langle\left(W_{0 \xi}\right)^{2}, e^{c \xi}\right\rangle_{\mathbf{R}}\left\langle K^{\kappa, 0}\left(\delta_{0}\right), \delta_{0}\right\rangle_{\mathbf{R}_{+}}\right. \\
& +\chi^{\prime}\left(\sigma^{*}\right)\left\{\left(K^{\kappa, 0}\left(\delta_{0}\right)\right)_{x}\left(\eta^{*}-0\right)\left\langle\left(W_{0 \xi}\right)^{2}, e^{c \xi}\right\rangle_{\mathbf{R}_{-}}\right. \\
& \left.\left.+\left(K^{\kappa, 0}\left(\delta_{0}\right)\right)_{x}\left(\eta^{*}+0\right)\left\langle\left(W_{0 \xi}\right)^{2}, e^{c \xi}\right\rangle_{\mathbf{R}_{+}}\right\}\right] .
\end{aligned}
$$

There exists a unique solution $\tau_{N}^{*}$ satisfying $\mathcal{F}\left(0, \tau_{N}^{*}, \kappa\right)=0$ and $\frac{\partial}{\partial \tau} \mathcal{F}\left(0, \tau_{N}^{*}, \kappa\right)=1$. Since $\mathcal{F}(\varepsilon, \tau, \kappa)$ is a real operator, there exists a solution $\tau=\tau(\varepsilon, \kappa) \in \mathbf{R}$ such that $\lim _{\varepsilon \downarrow 0} \tau(\varepsilon, \kappa)=\tau_{N}^{*}$ and $\mathcal{F}(\varepsilon, \tau(\varepsilon, \kappa), \kappa)=0$ for small $\varepsilon>0$. The above argument also holds for the Dirichlet boundary conditions.

On the other hand, in the case $\lim _{\varepsilon \downarrow 0} \varepsilon \kappa^{2}=\infty$, it easily follows from the above argument that $\lim _{\varepsilon \downarrow 0} \tau=-\infty$.

By using the same argument as given in [19], it is easy to show that these eigenvalues are simple. Therefore, the proof of Theorem 2 is completed.

Finally, we prove Lemma 6, that is, the principal eigenvalue $\zeta_{0}^{\varepsilon}$ of $T^{\varepsilon}$ is not an eigenvalue of $(5.4)_{0},(5.6)$. Assume that there exists a sequence $\varepsilon_{n}>0$ such that 
$\varepsilon_{n} \downarrow 0$ and $\zeta_{0}^{\varepsilon_{n}}$ is an eigenvalue. Let $\left(w_{n}, z_{n}\right)$ be the eigenfunction corresponding to the eigenvalue $\zeta_{0}^{\varepsilon_{n}}$, that is, $\left(T^{\varepsilon_{n}}-\zeta_{0}^{\varepsilon_{n}}\right) w_{n}=S^{\varepsilon_{n}}\left(\zeta_{0}^{\varepsilon_{n}}\right) z_{n}$. Since $\zeta_{0}^{\varepsilon_{n}}$ is the eigenvalue of $T^{\varepsilon_{n} *}$, we have

$$
\begin{aligned}
0 & =\left\langle w_{n},\left(T^{\varepsilon_{n} *}-\zeta_{0}^{\varepsilon_{n}}\right) \phi_{0}^{\varepsilon_{n} *}\right\rangle_{\mathbf{R}_{+}} \\
& =\left\langle\left(T^{\varepsilon_{n}}-\zeta_{0}^{\varepsilon_{n}}\right) w_{n}, \phi_{0}^{\varepsilon_{n} *}\right\rangle_{\mathbf{R}_{+}}=\left\langle S^{\varepsilon_{n}}\left(\zeta_{0}^{\varepsilon_{n}}\right) z_{n}, \phi_{0}^{\varepsilon_{n} *}\right\rangle_{\mathbf{R}_{+}} .
\end{aligned}
$$

Because $w_{n}=k_{n} \phi_{0}^{\varepsilon_{n}}+\left(T^{\varepsilon_{n}}-\zeta_{0}^{\varepsilon_{n}}\right)^{\dagger} S^{\varepsilon_{n}}\left(\zeta_{0}^{\varepsilon_{n}}\right) z_{n}$, it follows from (5.2)o that

$$
z_{n x x}+k_{n} \phi_{0}^{\varepsilon_{n}}+\left(T^{\varepsilon_{n}}-\zeta_{0}^{\varepsilon_{n}}\right)^{\dagger} S^{\varepsilon_{n}}\left(\zeta_{0}^{\varepsilon_{n}}\right) z_{n}-\gamma z_{n}=\zeta_{0}^{\varepsilon_{n}} z_{n}
$$

By using Lemma 10, we find that $z_{n}$ satisfies

$$
z_{n}=k_{n} K^{0, \zeta_{0}^{\varepsilon_{n}}} \phi_{0}^{\varepsilon_{n}}+K^{0, \zeta_{0}^{\varepsilon_{n}}}\left(T^{\varepsilon_{n}}-\zeta_{0}^{\varepsilon_{n}}\right)^{\dagger} S^{\varepsilon_{n}}\left(\zeta_{0}^{\varepsilon_{n}}\right) z_{n} .
$$

If $k_{n}=0$, then $z_{n}=K^{0, \zeta_{0}^{\varepsilon_{n}}}\left(T^{\varepsilon_{n}}-\zeta_{0}^{\varepsilon_{n}}\right)^{\dagger} S^{\varepsilon_{n}}\left(\zeta_{0}^{\varepsilon_{n}}\right) z_{n}$, which implies that $z_{n} \in H^{2}\left(\mathbf{R}_{+}\right)$. Since $\left\|K^{0, \zeta_{0}^{\varepsilon_{n}}}\left(T^{\varepsilon_{n}}-\zeta_{0}^{\varepsilon_{n}}\right)^{\dagger} S^{\varepsilon_{n}}\left(\zeta_{0}^{\varepsilon_{n}}\right)\right\|_{H^{2} \rightarrow H^{1}}=O(\varepsilon)$ for small $\varepsilon>0, z_{n}=0$ and $w_{n}=0$, which contradicts the assumption that $\left(w_{n}, z_{n}\right)$ is the eigenfunction. If $k_{n} \neq 0$, we set

$$
\begin{aligned}
& \hat{w}_{n}=\frac{w_{n}}{k_{n} \sqrt{\varepsilon_{n}}}=\frac{\phi_{0}^{\varepsilon_{n}}}{\sqrt{\varepsilon_{n}}}+\left(T^{\varepsilon_{n}}-\zeta_{0}^{\varepsilon_{n}}\right)^{\dagger} S^{\varepsilon_{n}}\left(\zeta_{0}^{\varepsilon_{n}}\right) \hat{z}_{n}, \\
& \hat{z}_{n}=\frac{z_{n}}{k_{n} \sqrt{\varepsilon_{n}}} .
\end{aligned}
$$

Therefore, (7.35) is rewritten as

$$
\hat{z}_{n x x}+\frac{\phi_{0}^{\varepsilon_{n}}}{\sqrt{\varepsilon_{n}}}+\left(T^{\varepsilon_{n}}-\zeta_{0}^{\varepsilon_{n}}\right)^{\dagger} S^{\varepsilon_{n}}\left(\zeta_{0}^{\varepsilon_{n}}\right) \hat{z}_{n}-\gamma \hat{z}_{n}=\zeta_{0}^{\varepsilon_{n}} \hat{z}_{n}
$$

It follows from (7.34) that

$$
\begin{aligned}
0= & \lim _{n \rightarrow \infty}\left\langle S^{\varepsilon_{n}}\left(\zeta_{0}^{\varepsilon_{n}}\right) \hat{z}_{n}, \frac{\phi_{0}^{\varepsilon_{n} *}}{\sqrt{\varepsilon_{n}}}\right\rangle_{\mathbf{R}_{+}}=-k \gamma^{*} \kappa^{*}\left\{\chi^{\prime \prime}\left(\sigma^{*}\right) v_{x}^{*}\left\langle\hat{z}_{n}, \delta_{0}\right\rangle_{\mathbf{R}_{+}}\left\langle\left(W_{0 \xi}\right)^{2}, e^{c \xi}\right\rangle_{\mathbf{R}}\right. \\
& \left.+\chi^{\prime}\left(\sigma^{*}\right)\left[\hat{z}_{n x}\left(\eta^{*}-0\right)\left\langle\left(W_{0 \xi}\right)^{2}, e^{c \xi}\right\rangle_{\mathbf{R}_{-}}+\hat{z}_{n x}\left(\eta^{*}+0\right)\left\langle\left(W_{0 \xi}\right)^{2}, e^{c \xi}\right\rangle_{\mathbf{R}_{+}}\right]\right\} .
\end{aligned}
$$

Hence, we have

$$
\begin{aligned}
0=\chi^{\prime \prime}\left(\sigma^{*}\right) v_{x}^{*}\left\langle\hat{z}_{n}, \delta_{0}\right\rangle_{\mathbf{R}_{+}}\left\langle\left(W_{0 \xi}\right)^{2}, e^{c \xi}\right\rangle_{\mathbf{R}} \\
\quad+\chi^{\prime}\left(\sigma^{*}\right)\left[\hat{z}_{n x}\left(\eta^{*}-0\right)\left\langle\left(W_{0 \xi}\right)^{2}, e^{c \xi}\right\rangle_{\mathbf{R}_{-}}+\hat{z}_{n x}\left(\eta^{*}+0\right)\left\langle\left(W_{0 \xi}\right)^{2}, e^{c \xi}\right\rangle_{\mathbf{R}_{+}}\right] .
\end{aligned}
$$

From (7.36), $\hat{z}_{n}$ satisfies

$$
\begin{aligned}
\zeta_{0}^{\varepsilon_{n}}\left\langle\hat{z}_{n}, \psi\right\rangle_{\mathbf{R}_{+}}=- & \left\langle\hat{z}_{n x}, \psi_{x}\right\rangle_{\mathbf{R}_{+}}+\left\langle\frac{\phi_{0}^{\varepsilon_{n}}}{\sqrt{\varepsilon_{n}}}, \psi\right\rangle_{\mathbf{R}_{+}} \\
& +\left\langle\left(T^{\varepsilon_{n}}-\zeta_{0}^{\varepsilon_{n}}\right)^{\dagger} S^{\varepsilon_{n}}\left(\zeta_{0}^{\varepsilon_{n}}\right) \hat{z}_{n}, \psi\right\rangle_{\mathbf{R}_{+}}-\gamma\left\langle\hat{z}_{n}, \psi\right\rangle_{\mathbf{R}_{+}}
\end{aligned}
$$

for each $\psi \in H_{N}^{1}\left(\mathbf{R}_{+}\right)$. Since $\lim _{n \rightarrow \infty} \hat{z}_{n}=\hat{z}^{*}$ in $H_{N}^{1}\left(\mathbf{R}_{+}\right)$, it follows that

$$
0=-\left\langle\hat{z}_{x}^{*}, \psi_{x}\right\rangle_{\mathbf{R}_{+}}+\kappa^{*}\left\langle\delta_{0}, \psi\right\rangle_{\mathbf{R}_{+}}-\gamma\left\langle z^{*}, \psi\right\rangle_{\mathbf{R}_{+}}
$$

by Lemma 11 . The solution $z^{*}$ of (7.38) is represented by

$$
\hat{z}^{*}(x)= \begin{cases}a \frac{e^{\sqrt{\gamma} x}+e^{-\sqrt{\gamma} x}}{e^{\sqrt{\gamma} \eta^{*}}+e^{-\sqrt{\gamma} \eta^{*}}}, & x \in\left(0, \eta^{*}\right), \\ a e^{-\sqrt{\gamma}\left(x-\eta^{*}\right)}, & x \in\left(\eta^{*}, \infty\right),\end{cases}
$$


where $\left\langle z^{*}, \delta_{0}\right\rangle_{\mathbf{R}_{+}}=a$. It follows from (7.37) and (7.39) that

$$
\begin{aligned}
0=a & \sqrt{\gamma}\left[\chi^{\prime}\left(\sigma^{*}\right)\left\{\frac{1-e^{-2 \sqrt{\gamma} \eta^{*}}}{1+e^{-2 \sqrt{\gamma} \eta^{*}}}\left\langle\left(W_{0 \xi}\right)^{2}, e^{c \xi}\right\rangle_{\mathbf{R}_{-}}-\left\langle\left(W_{0 \xi}\right)^{2}, e^{c \xi}\right\rangle_{\mathbf{R}_{+}}\right\}\right. \\
& \left.-\chi^{\prime \prime}\left(\sigma^{*}\right) \sigma^{*}\left\langle\left(W_{0 \xi}\right)^{2}, e^{c \xi}\right\rangle_{\mathbf{R}}\right] .
\end{aligned}
$$

By Lemma 4, we have $a=0$, that is, $\hat{z}^{*}(x)=0$. This contradicts (7.38), so that the lemma is proved for $(5.4)_{0},(5.6)_{N}$. By using the above argument, Lemma 11 also holds for $(5.4)_{0},(5.6)_{D}$. Thus, $\zeta_{0}^{\varepsilon_{n}}$ is not an eigenvalue of $(5.4)_{0},(5.6)$, so Lemma 6 is proved.

\section{Concluding remarks}

We have shown that the location of critical eigenvalues of the linearized problem around the symmetric planar equilibrium solutions $\left(u^{\varepsilon}, v^{\varepsilon}\right)$ for $(1.2)-(1.5)$ in the strip domain is determined by the eigenvalues corresponding to the singular limit eigenvalue problem obtained as $\varepsilon \downarrow 0$. Particularly in the 1-dimensional symmetric equilibrium solution, Corollary 1 implies that stability is determined by the sign of $\frac{d}{d \eta} H\left(\eta^{*}\right)$. We emphasize that there is a close relation between the stability condition in the 1-dimensional domain and the matching condition (7.15) of the singular perturbation method, which already was pointed out for reaction-diffusion systems [12, 20]. Theorem 2 says that the analysis of the interface equation, which is called the singular limit analysis, is essential to show the stability of the equilibrium solution of (1.2)-(1.5) with small $\varepsilon>0$.

In this paper, we have considered the equilibrium solutions of (1.2)-(1.5) in the strip domain. We also know that for any $k>0$, there is a planar traveling front solution under the boundary condition

$$
\lim _{x \rightarrow-\infty}(u(\tau, x, y), v(\tau, x, y))=\left(1, \frac{1}{\gamma}\right) \quad \text { and } \quad \lim _{x \rightarrow \infty}(u(\tau, x, y), v(\tau, x, y))=(0,0)
$$

where $(1,1 / \gamma)$ and $(0,0)$ are stable constant equilibrium solutions of (1.2). The existence and stability of planar traveling front solution will be shown in a forthcoming paper [17].

The existence and stability of radially symmetric localized equilibrium solutions in the whole domain $\mathbf{R}^{2}$ with the boundary condition

$$
\lim _{|(x, y)| \rightarrow \infty}(u(\tau, x, y), v(\tau, x, y))=(0,0)
$$

are also interesting problems. It is shown in [15], [16] that the stability is revealed by solving the linearized problem of the limiting system. Numerical simulation suggests that the theorem corresponding to Theorem 2 holds, but this remains unsolved.

Acknowledgement. The author would like to thank Professor Masayasu Mimura of University of Tokyo for his consistently valuable advice and encouraging support for completing this work.

\section{References}

1. J. Adler, Chemotaxis in bacteria, Science 153 (1966), 708-716.

2. H. C. Berg and L. Turner, Chemotaxis of bacteria in glass capillary, Biophys. J. 58 (1990), 919-930.

3. E. O. Budrene and H. C. Berg, Complex patterns formed by motile cells of Escherichia coli, Nature 349 (1991), 630-633. 
4. X. Chen, Generation and propagation of interfaces for reaction-diffusion equation, J. Differential Equations 96 (1992), 116-141.

5. E. A. Coddington and N. Levinson, Theory of Ordinary Differential Equations, McGraw-Hill, New York, 1955.

6. F. W. Dalquist, P. Lovely, and D. E. Koshland, Jr., Quantitative analysis of bacterial migration in chemotaxis, Nature New Biol. 236 (1972), 120-123.

7. P. C. Fife, Boundary and interior transition layer phenomena for pairs of second-order differential equations, J. Math. Anal. Appl. 54 (1976), 497-521.

8. R. M. Ford and D. A. Lauffenburger, Analysis of chemotactic bacterial distributions in population migration assays using a mathematical model applicable to steep or shallow attractant gradients, Bull. Math. Biol. 53 (1991), 721-749.

9. Y. Furuya and A. Yagi, Linearized stability for abstract quasilinear evolution equations of parabolic type II, time non homogeneous case, Advances in Math. Sci. and Appl. 3 (1993), 285-300.

10. D. Henry, Geometric Theory of Semilinear Parabolic Equations, Lecture Notes in Mathematics 80, Springer-Verlag, Berlin, New York, 1981.

11. Y. Hosono and M. Mimura, Singular perturbation approach to traveling waves in competing and diffusing species models, J. Math. Kyoto Univ. 22 (1982), 435-461.

12. H. Ikeda, M. Mimura, and T. Tsujikawa, Singular perturbation approach to traveling waves solutions of the Hodgkin-Huxley equations and its application to stability problems, Japan J. Appl. Math. 6 (1989), 1-66.

13. E. F. Keller and L. A. Segel, Initiation of slime mold aggregation viewed as instability, J. Theor. Biol. 26 (1970), 399-415.

14. M. Mimura, M. Tabata, and Y. Hosono, Multiple solutions of two-point boundary-value problems of Neumann type with a small parameter, SIAM J. Math. Anal. 13 (1982), 555-593.

15. M. Mimura and T. Tsujikawa, Aggregating pattern dynamics in a chemotaxis model including growth, Physica A 230 (1996), 499-543.

16. M. Mimura, T. Tsujikawa, R. Kobayashi, and D. Ueyama, Dynamics of aggregating patterns in a chemotaxis-diffusion-growth model, Forma 8 (1993), 179-195.

17. M. Mimura, T. Tsujikawa, and M. Funaki, in preparation

18. J. D. Murray, Mathematical Biology, Springer-Verlag, Berlin Heidelberg, 1989.

19. Y. Nishiura and H. Fujii, Stability of singularly perturbed solutions to systems of reactiondiffusion equations, SIAM J. Math. Anal. 18 (1987), 1726-1770.

20. Y. Nishiura, M. Mimura, H. Ikeda, and H. Fujii, Singular limit analysis of stability of traveling wave solutions in bistable reaction-diffusion systems, SIAM J. Math. Anal. 21 (1990), 85-122.

21. T. Ohta, M. Mimura, and R. Kobayashi, Higher-dimensional localized patterns in excitable media, Physica D 34 (1989), 115-144.

22. M. Potier-Ferry, The linealization principle for the stability of solutions of quasilinear parabolic equations I, Arch. Rational Mech. Anal. 77 (1981), 301-320.

23. D. H. Sattinger, On the stability of waves of nonlinear parabolic systems, Advances in Math. 22 (1976), 312-355.

24. M. Taniguchi, Bifurcation from flat-layered solutions to reaction-diffusion systems in two space dimensions, J. Math. Sci. Univ. Tokyo. 1 (1994), 339-367.

25. A. Yagi, M. Mimura, and T. Tsujikawa, in preparation.

Faculty of Engineering, Hiroshima Denki Institute of Technology, Hiroshima 739-03, Japan 